\title{
On linear normal lattices configurations
}

\author{
par MoRdechay B. LEVIN et MEIR SMORODINSKY
}

RÉSUMÉ. Dans cet article nous prolongeons la construction de Champernowne de nombres normaux dans la base $b$ pour le cas $\mathbb{Z}^{d}$, et obtenons une construction explicite du point générique de la transformation de l'ensemble $\{0,1, \ldots, b-1\}^{\mathbb{Z}^{d}}$ par $\mathbb{Z}^{d}$ déplacement. Nous prouvons que l'intersection de la configuration de réseau considérée avec une droite arbitraire est une suite normale dans la base $b$.

Abstract. In this paper we extend Champernowne's construction of normal numbers in base $b$ to the $\mathbb{Z}^{d}$ case and obtain an explicit construction of the generic point of the $\mathbb{Z}^{d}$ shift transformation of the set $\{0,1, \ldots, b-1\}^{\mathbb{Z}^{d}}$. We prove that the intersection of the considered lattice configuration with an arbitrary line is a normal sequence in base $b$.

\section{Introduction}

A number $\alpha \in(0,1)$ is said to be normal to base $b$, if in the $b$-ary expansion of $\alpha, \alpha=d_{1} d_{2} \cdots \quad\left(d_{i} \in\{0,1, \cdots, b-1\}, i=1,2, \cdots\right)$, each fixed finite block of digits of length $k$ appears with an asymptotic frequency of $b^{-k}$ along the sequence $\left(d_{i}\right)_{i \geq 1}$. Normal numbers were introduced by Borel (1909). Champernowne [2] gave an explicit construction of such a number, namely,

$$
\theta=.123456789101112 \ldots,
$$

obtained by successively concatenating the digital expansions all the natural numbers.

We shall call the sequence of digits obtained from a normal number a "normal sequence".

Champernowne's construction is associated with the independent identical distributed process of variables having uniform distribution over $b$ states. In [1], [14], and [15], constructions of normal sequences for various stationary stochastic processes, similar to Champernowne's were introduced.

Manuscrit reçu le ler février 2004.

Work supported in part by the Israel Science Foundation Grant No. 366-172. 
Our goal is to extend such constructions to $\mathbb{Z}^{d}$-arrays $(d>1)$ of random variables, which we shall call $\mathbb{Z}^{d}$-processes. We shall deal with stationary $\mathbb{Z}^{d}$-processes, that is, processes with distribution, invariant under the $\mathbb{Z}^{d}$ action. We shall call a specific realization of a $\mathbb{Z}^{d}$-process a "configuration" ("lattice configuration"). To begin with, the very definition of a "normal configuration" is subject to various generalizations from the 1-dimensional case. In this paper we continue investigations beginning in [11] and [12] (see also [3] and [8]).

Rectangular normality. We denote by $\mathbb{N}$ the set of non-negative integers. Let $d, b \geq 2$ be two integers, $\mathbb{N}^{d}=\left\{\left(n_{1}, \ldots, n_{d}\right) \mid n_{i} \in \mathbb{N}, i=1, \ldots, d\right\}$, $\Delta_{b}=\{0,1, \ldots, b-1\}, \Omega=\Delta_{b}^{\mathbb{N}^{d}}$.

We shall call $\omega \in \Omega$ a configuration (lattice configuration). A configuration is a function $\omega: \mathbb{N}^{d} \rightarrow \Delta_{b}$.

Given a subset $F$ of $\mathbb{N}^{d}, \omega_{F}$ will be the restriction of the function $\omega$ to $F$. Let $\mathbf{N} \in \mathbb{N}^{d}, \mathbf{N}=\left(N_{1}, \ldots, N_{d}\right)$. We denote a rectangular block by

$$
F_{\mathbf{N}}=\left\{\left(f_{1}, \ldots, f_{d}\right) \in \mathbb{N}^{d} \mid 0 \leq f_{i}<N_{i}, i=1, \ldots, d\right\},
$$

$\mathbf{h}=\left[0, h_{1}\right) \times \ldots \times\left[0, h_{d}\right), h_{i} \geq 1, i=1, \ldots, d ; G=G_{\mathbf{h}}$ is a fixed block of digits $G=\left(g_{\mathbf{i}}\right)_{\mathbf{i} \in F_{\mathbf{h}}}, \quad g_{\mathbf{i}} \in \Delta_{b} ; \quad \chi_{\omega, G}(\mathbf{f})$ is a characteristic function of the block of digits $G$ shifted by vector $\mathbf{f}$ in the configuration $\omega$

$$
\chi_{\omega, G}(\mathbf{f})=\left\{\begin{array}{l}
1 \quad \text { if } \omega(\mathbf{f}+\mathbf{i})=g_{\mathbf{i}}, \quad \forall \mathbf{i} \in F_{\mathbf{h}} \\
0 \quad \text { otherwise }
\end{array}\right.
$$

Definition 1. $\omega \in \Omega$ is said to be rectangular normal if for any $\mathbf{h} \subset \mathbb{N}^{d}$ and block $G_{\mathbf{h}}$

$$
\#\left\{\mathbf{f} \in F_{\mathbf{N}} \mid \chi_{\omega, G_{\mathbf{h}}}(\mathbf{f})=1\right\}-b^{-h_{1} \ldots h_{d}} N_{1} \ldots N_{d}=o\left(N_{1} \ldots N_{d}\right),
$$

when $\max \left(N_{1}, \ldots, N_{d}\right) \rightarrow \infty$.

We shall say that $\omega$ is square normal in case we consider only square blocks i.e., $N_{1}=N_{2}=\ldots=N_{d}$. For the sake of clarity, we shall carry the proof only for the case $d=2$.

Linear normality. Let

$$
\omega=\left\{f_{i j} \mid f_{i j} \in \Delta_{b}, \quad i, j=0,1,2, \ldots\right\}
$$

be a 2 -dimensional configuration; $\alpha \geq 0, \beta$ be real. Let the tiling of the plane by unit squares be given. We label the squares of the tiles of the positive quadrant of the plane by $\omega_{i j}$ where $(i, j)$ are the coordinates of the left, lower vertex of the tile. Consider the line $y=\alpha x+\beta$ (or the vertical line $x=\beta$ ). The line is partitioned into successive intervals of the intersections with tiles. Therefore, to each line corresponds a sequence of digits

$$
\left(u_{\alpha, \beta}(n)\right)_{n \geq 0} .
$$


Let

(3)

$$
R_{N}(\omega, \alpha, \beta, m)=\#\left\{0 \leq n<N \mid u_{\alpha, \beta}(n+i)=g_{i}, i=1, \ldots, m\right\}-b^{-m} N
$$

Definition 2. $\omega$ is said to be linear normal if for all reals $\alpha \geq 0, \beta$ the sequence $\left(u_{\alpha, \beta}(n)\right)_{n \geq 0}$ is normal in base b, i.e.,

$$
R_{N}(\omega, \alpha, \beta, m)=o(N)
$$

for all integer $s \geq 1$ and all blocks of digits $\left(g_{1}, \ldots, g_{m}\right) \quad\left(g_{i} \in \Delta_{b}, i=\right.$ $1, \ldots, m)$.

Construction. Let

$$
L\left(f_{1}, f_{2}\right)= \begin{cases}f_{1}^{2}+f_{2}, & \text { if } f_{2}<f_{1} \\ f_{2}^{2}+2 f_{2}-f_{1} & \text { if } f_{2} \geq f_{1}\end{cases}
$$

This map is clearly a bijection between $\mathbb{N}$ and $\mathbb{N}^{2}$, inducing a total order on $\mathbb{N}^{2}$ from the usual one on $\mathbb{N}$. We define the configuration $\omega_{n}$ on $F_{\left(2 n b^{2 n^{2}}, 2 n b^{2 n^{2}}\right)}$ as the concatenation of $b^{4 n^{2}}$ blocks of digits of size $2 n \times 2 n$ with the left lower corner $(2 n x, 2 n y), \quad 0 \leq x, y<b^{2 n^{2}}$. To each of these blocks we set in correspondence the number $L(x, y)$. Next we use $b$-expansion of the number $L(x, y)$ according to the order $L$ to obtain digits of the considered $2 n \times 2 n$ block. It is easy to obtain the analytic expression for digits of the configuration $\omega_{n}$ :

$$
\omega_{n}(2 n x+s, 2 n y+t)= \begin{cases}a_{s^{2}+t}(u), & \text { if } t<s \\ a_{t^{2}+2 t-s}(u) & \text { if } t \geq s\end{cases}
$$

where

$$
u=u(x, y)= \begin{cases}x^{2}+y, & \text { if } y<x \\ y^{2}+2 y-x & \text { if } y \geq x,\end{cases}
$$

$s, t, x, y$ are integers, $0 \leq x, y<b^{2 n^{2}}, 0 \leq s, t<2 n$, and

$$
n=\sum_{i \geq 0} a_{i}(n) b^{i}, \quad\left(a_{i}(n) \in\{0,1, \ldots, b-1\}\right)
$$

is the $b$-expansion of integer $n$.

Next we define inductively a sequence of increasing configurations $\omega_{n}$ on

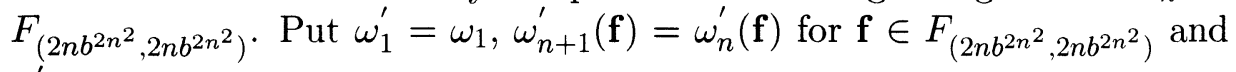
$\omega_{n+1}^{\prime}(\mathbf{f})=\omega_{n+1}(\mathbf{f})$ otherwise. Put

$$
\begin{aligned}
& \omega_{\infty}=\lim \omega_{n}^{\prime}, \\
& \left(\omega_{\infty}\right)_{F_{\left(2 n b^{2 n^{2}} .2 n b^{2 n^{2}}\right)}}=\omega_{n}^{\prime}, \quad n=1,2, \ldots .
\end{aligned}
$$


Theorem 1. $\omega_{\infty}$ is a rectangular and linear normal configuration with

$$
R_{N}\left(\omega_{\infty}, \alpha, \beta, m\right)=O\left(N /(\log N)^{1 / 4}\right)
$$

with $O$-constant depending on $\alpha, \beta$, and $m$.

The rectangular normality of $\omega_{\infty}$ follows from [11] or [12]. Proof of the linear normality property is given in Section 3 .

Theorem 2 Let $\mu$ be a Bernoulli measure on $\Omega$, then $\mu$-almost all $\omega$ are linear normal.

Proof. We consider first the lines with $0 \leq \beta<1$ and the finite sequences that are determined by these lines confined to the square $\{(x, y): 0 \leq$ $x, y \leq N\}$. There are at most $3 N^{2}$ different finite sequences $\left(u_{\alpha, \beta}(n)\right)_{n \geq 0}$ whose length can vary between $N-1$ and $2 N$. Given $\epsilon>0$, (using the Chebishev inequality or the central limit theorem) the $\mu$ measure of the average along any of these sequences to exceed the expected value by more than $\epsilon$ is less than $C / N^{4}$. Using Borel-Cantelli will prove the claim for these lines. To conclude the claim for lines of all values of $\beta$, observe that $\mu$ is invariant under translations.

\section{Generalizations, examples, and problems.}

\subsection{Polynomial normality.}

Let

$$
\omega=\left\{g_{i j} \mid g_{i j} \in \Delta_{b}=\{0,1, \ldots, b-1\}, i, j=0,1,2, \ldots\right\}
$$

be a 2 -dimensional configuration; $\alpha_{i}(i=0,1, \ldots, d)$ be reals, $\phi(x)=\alpha_{d} x^{d}+$ $\ldots+\alpha_{1} x+\alpha_{0}, \alpha_{d}>0$. Let the tiling of the plane by unit squares be given. We label the squares of the tiles of the positive quadrant of the plane by $\omega_{i j}$ where $(i, j)$ are the coordinates of the left, lower vertex of the tile. Consider the curve $y=\phi(x)$ (or the curve $x=\phi(y)$ ). The curve is partitioned into successive intervals of the intersections with tiles. Therefore, to each curve corresponds a sequence of digits

$$
\left(u_{\phi}(n)\right)_{n \geq 0}
$$

Definition 3. The configuration $\omega$ is said to be polynomial normal if for all real polynomial $\phi$ the sequence $\left(u_{\phi}(n)\right)_{n \geq 0}$ is normal in base $b$.

In [13] we proved that the configuration $\omega_{\infty}$ is polynomial normal.

Now we note that notions of linear, polynomial, square, and rectangular normal configurations define different sets in $\Omega$. These differences have null measure subsets, but are not empty:

Example 1. A configuration which is linear normal, but not square normal: Let $x(n)$ be an arbitrary normal sequence in base $b$, put $\omega(i, j)=$ $x(i+j), i, j=1,2, \ldots$. This is not a square normal configuration since the $2 \times 2$ block of digits $\left(\begin{array}{l}10 \\ 00\end{array}\right)$ in $\omega$ does not appear in this configuration. 
Bearing in mind that $\omega(i, j)=\omega(j, i)$, we obtain it is sufficient to consider only the case $0 \leq \alpha \leq 1$. It is easy to see that $u_{\alpha, \beta}(n)=x(f(n))$ with $f(n) \in[n+[\alpha n+\beta], n+[\alpha(n+1)+\beta]]$. Observe that $(f(n))_{n \leq 0}$ is a completely deterministic sequence in the sense of B. Weiss [16] (see also [5, p. 147-148]) and thus $\left(u_{\alpha, \beta}(n)\right)_{n \geq 0}$ is a normal sequence.

Example 2. A configuration which is rectangle normal, but not linear normal: Given $\omega$ is a rectangle normal configuration, change the configuration so that $\omega^{\prime}(i, i)=0, i,=1,2, \ldots$.

Example 3. A configuration which is rectangle and linear normal, but not polynomial normal: Similar to Example 2. Change the configuration along non-linear polynomial line.

Example 4. A configuration which is square and linear normal, but not rectangular normal:

Construction: Again we begin by constructing for each natural $n$, a block configuration $\left.\omega_{n}(u, v)\right)_{0 \leq u, v<2 n b^{2 n^{2}}}$. We put

$$
\left.\omega_{n}(2 n x+s, 2 n y+t)\right)= \begin{cases}a_{2 n t+s}(x), & \text { if } t<n \\ a_{n s+t-n}(y) & \text { if } t \geq n,\end{cases}
$$

where $s, t, x, y$ are integers, $0 \leq x, y<b^{2 n^{2}}, 0 \leq s, t<2 n$.

Let us describe the structure of the configuration $\omega_{n}$ in words. $\omega_{n}$ is composed of configurations of $2 n \times 2 n$ blocks. Each such block is composed of two $2 n \times n$ lower and upper rectangles. Each rectangle can store the expansion of an integer $m, \quad 0 \leq m<b^{2 n^{2}}$. Given a number $m$, we store the digits in lower rectangles by first using the bottom row (left to right) then going to the next upper row etc.

For each row of bottom rectangles we store all the different $b^{2 n^{2}}$ numbers in their natural order. So all the rows of bottom rectangles look the same. For the upper rectangles we use a different rule to store a number $m$. We first use the first column (from top to bottom) then the second row etc. We proceed by storing for each column of top rectangles all the different numbers. Notice that at the end if we observe the $2 n \times 2 n$ configurations of $\omega_{n}$ all the possible configurations appear.

This choice of $\omega_{n}$ will lead to a configuration that will guarantee the normality of all lines $y=\alpha x+\beta$. To guarantee the normality of all vertical lines too, we shall have to introduce a variant of the definition of $\omega_{n}$. Namely: apply (9) for all $x>0$ or $x=0$ and $s>n$; for $x=0$ and $s \in[0, n)$ use

$$
\left.\omega_{n}(s, 2 n y+t)\right)=a_{2 n s+t}(y)
$$

instead of (2), where $0 \leq y<b^{2 n^{2}}, 0 \leq t<2 n$. We now proceed with the construction of the desired configuration. We construct the sequence of 
block configurations (in a similar way as in the first construction see (8)) inductively.

These considerations lead to the following problems:

Problem 1. Is the intersection of $\omega_{\infty}$ with all monotone, increasing convex curves also normal?

Problem 2. Is the Davenport-Erdös like rectangular normal configuration from [11] also linear (polynomial) normal?

\section{2. $s$-dimensional surfaces in $\mathbb{R}^{d}$.}

Consider a function $\psi: \mathbb{R}^{s} \rightarrow \mathbb{R}^{d}$. Let $G_{\psi}=\left\{\psi(\mathbf{x}) \in \mathbb{R}^{d} \mid \mathbf{x} \in \mathbb{R}^{s}\right\}$, $s \leq d$,

$$
G_{\psi}^{\prime}=\left\{\mathbf{n} \in \mathbb{Z}^{d} \mid \mathbf{n}+[0,1)^{d} \cap G_{\psi} \neq \emptyset\right\}, \quad H_{\psi}: G_{\psi}^{\prime} \rightarrow \mathbb{Z}^{s},
$$

and $\Psi=\{\psi\}$ is a set of function $\psi$ (a set of $s$-dimensional surfaces), such that $H_{\psi}$ is a bijection with $H_{\psi}\left(G_{\psi}^{\prime}\right)=\mathbb{Z}^{s}$.

Definition 4. The configuration $\omega \in\{0,1, \ldots, b-1\}^{\mathbb{Z}^{d}}$ is said to be $\Psi$ normal if $\omega\left(G_{\psi}^{\prime}\left(H_{\psi}^{-1}\right)\right)$ is rectangular normal in $\mathbb{Z}^{s}$ for all $\psi \in \Psi$.

Problem 3. Let $\omega$ be a $d$-dimensional configuration, constructed similarly to (3) - (7); $\Psi_{p}$ be a set of all $s$-dimensional polynomial surfaces in $\mathbb{R}^{d}$. Is $\omega$ a $\Psi_{p}$-normal configuration?

\subsection{Connection with uniform distribution.}

Let $\left(\mathbf{x}_{\mathbf{n}}\right)_{\mathbf{n} \geq 1}$ be an infinite sequence of points in an $s$-dimensional unit cube $[0,1)^{s}$; let $v=\left[0, \gamma_{1}\right) \times \ldots \times\left[0, \gamma_{s}\right)$ be a box in $[0,1)^{s}$; and $A_{v}(N)$ be the number of indexes $n \in[1, N]$ such that $\mathbf{x}_{n}$ lies in $v$. The quantity

$$
D_{N}=D\left(\left(\mathbf{x}_{n}\right)_{n=1}^{N}\right)=\sup _{v \in(0,1]^{s}}\left|\frac{1}{N} A_{v}(N)-\gamma_{1} \ldots \gamma_{s}\right|
$$

is called the discrepancy of $\left(\mathbf{x}_{\mathbf{n}}\right)_{\mathbf{n}=1}^{\mathbf{N}}$. The sequence $\left(\mathbf{x}_{\mathbf{n}}\right)_{\mathbf{n} \geq \mathbf{1}}$ is said to be uniformly distributed in $[0,1)^{s}$ if $D_{N} \rightarrow 0$.

It is known (Wall, 1949, see [7, p. 70]), that a number $\alpha$ is normal to base $b$ if and only if the sequence $\left\{\alpha b^{n}\right\}_{n \geq 1}$ is uniformly distributed (abbreviated u.d.) in $[0,1)$.

Let $\omega=\left(a_{i, j}\right)_{i, j \geq 1} \quad\left(a_{i, j} \in\{0,1, \ldots, b-1\}\right)$ be a configuration

$$
\alpha_{m}=\sum_{i=1}^{\infty} a_{m, i} / b^{i}, \quad m=1,2, \ldots,
$$

$s \geq 1$ be an integer. The following statement is proved in [9]: 
The lattice configuration $\omega$ is normal to base $b$ if and only if for all $s \geq 1$ the double sequence

$$
\left(\left\{\alpha_{m} b^{n}\right\}, \ldots,\left\{\alpha_{m+s-1} b^{n}\right\}\right)_{m, n \geq 1}
$$

is uniformly distributed in $[0,1)^{s}$, i.e.,

$$
D\left(\left(\left\{\alpha_{m} b^{n}\right\}, \ldots,\left\{\alpha_{m+s-1} b^{n}\right\}\right)_{1 \leq n \leq N, 0 \leq m<M}\right)=o(1),
$$

when $\max (M, N) \rightarrow \infty$. Hence we have another definition of normal configuration (of normal sequence $\alpha=\left(\alpha_{1}, \alpha_{2}, \ldots\right) \in[0,1)^{\infty}$ to the base $b$ ). It is evident that a.e. sequences $\alpha$ are normal for all bases $b \geq 2$ (absolutely normal). It is easy to see that the u.d. definition of normality permits to transport a large set of problems on normal numbers to the $\mathbb{Z}^{d}$ case, for example, normality in different bases. Another example: in [9] we prove that the lower bound of the discrepancy of the double sequence (11)-(12) coincides (with the exactitude of the logarithmic multiplier) with the lower bound of the discrepancy of ordinary sequences $\left(x_{n}\right)_{n=1}^{M N}$ in an $s$-dimensional unit cube $(s, M, N=1,2, \ldots)$.

\subsection{Connection with completely uniform distribution.}

Now let $\left(v_{n}\right)_{n \geq 1}$ be an arbitrary sequence of real numbers. Starting with the sequence $\left(v_{n}\right)_{n \geq 1}$, we construct for every integer $s \geq 1$ the $s$-dimensional sequence $\left(x_{n}^{(s)}\right)=\left(\left\{v_{n+1}\right\}, \ldots,\left\{v_{n+s}\right\}\right)$, where $\{x\}$ is the fractional part of $x$. The sequence $\left(v_{n}\right)_{n \geq 1}$ is said to be completely uniformly distributed (abbreviated c.u.d.) if for any integer $s \geq 1$ the sequence $\left(x_{n}^{(s)}\right)$ is u.d. in $[0,1)^{s}$ (Korobov, 1949, see [6]). The c.u.d. sequence is the universal sequence for computing multidimensional integrals, modeling Markov chains, random numbers, and for other problems $[4,6,7]$. Let $b \geq 2$ be an integer, $\left(v_{n}\right)$ be a c.u.d. sequence, $a_{n}=\left[b\left\{v_{n}\right\}\right], n=1,2, \ldots$. Then $\alpha=._{1} a_{2} \ldots$ is normal to base $b$ (Korobov, [6]). In [10] we constructed a c.u.d. double sequence $\left(v_{n, m}\right)_{n, m \geq 1}$, such that for all integers $s, t \geq 1$

$$
M N D\left(\left(\left(v_{n+i, m+j}\right)_{i=1, j=1}^{s, t}\right)_{n=1, m=1}^{N, M}\right)=O\left((\log (M N+1))^{s t+4}\right)
$$

for all $M, N \geq 1$. Similarly to [6], we get from here the estimate of error term in (2) as $O\left(\left(\log \left(N_{1} N_{2}+1\right)\right)^{s t+4}\right)$ for the configuration $\left(a_{n, m}\right)_{n, m \geq 1}$, where $a_{n, m}=\left[b\left\{v_{n, m}\right\}\right], n, m \geq 1$.

Let $\phi=\{x, y \mid x, y \in \mathbb{R}, y=\phi(x)\}$ be a curve in the plane. Define a sequence of reals by $\left(v_{\phi}(n)\right)_{n \geq 1}=\left\{v_{i, j} \mid(i, j) \in \mathbb{N}^{d},(i, j)+[0,1)^{2} \cap \phi \neq \emptyset\right\}$.

Problem 4. Find explicitly the c.u.d. double sequence $\left(v_{n, m}\right)_{n, m \geq 1}$, such that the sequence $\left(v_{\phi}(n)\right)_{n \geq 1}$ is c.u.d. for all linear (polynomial) curves $\phi$. 


\section{Auxiliary notations and results.}

To estimate the discrepancy we use the Erdös-Turan-Koksma inequality (see, for example, [4] p.18)

$$
N D_{N} \leq\left(\frac{3}{2}\right)^{k}\left(\frac{2 N}{M+1}+\sum_{0<\max _{1 \leq i \leq k}\left(\left|m_{\imath}\right|\right) \leq M} \frac{\left|\sum_{x=0}^{N-1} e\left(m_{1} u_{1 x}+\ldots+m_{k} u_{k x}\right)\right|}{\bar{m}_{1} \ldots \bar{m}_{k}}\right)
$$

where $e(y)=e^{2 \pi i y}, \bar{m}=\max (1,|m|), \quad M \geq 1$ - arbitrary.

We shall use the following estimates (see, for example, [6] p.1)

$$
\left|\sum_{x=A}^{A+q-1} e(\theta x)\right| \leq \min \left(P, \frac{1}{2\|\theta\|}\right),
$$

where $\|\theta\|=\min (\{\theta\}, 1-\{\theta\})$.

\section{Proof of Theorem 1.}

We consider separately the following cases: when the line is horizontal, vertical, rational $(\alpha \in \mathbb{Q})$, and irrational $(\alpha \in \mathbb{R} \backslash \mathbb{Q})$. We start with the difficult case:

\subsection{The case of irrational $\alpha$.}

We shall find the number of occurences of a fixed block of digits in the considered configuration in term of Diophantine inequalities.

Since $\alpha$ is irrational, $\{\alpha i\} \neq\{\alpha j\} \quad(i \neq j)$. Let $\left(\xi_{i}\right)_{i=1}^{m}$ be the set $\{1-\{\alpha i\} \mid i=1, \ldots, m\}$ arranged in increasing order. Put $\xi_{0}=0, \xi_{m+1}=1$. Let $f$ be the map of the set $\{1, \ldots, m\}$ so that

$$
\xi_{f(i)}=1-\{\alpha i\}, i=1, \ldots, m .
$$

For a fixed integer $\nu \in[0, m]$ consider the sequence $\left(l_{i}(\nu)\right)_{i=1}^{m}$ :

$$
l_{i}(\nu)= \begin{cases}{[\alpha i],} & \text { if } \nu<f(i) \\ {[\alpha i]+1} & \text { otherwise }\end{cases}
$$

Lemma 1. Let

$$
\left\{\frac{\alpha(2 n x+s)+\beta}{2 n}\right\} \in\left[\frac{t+\xi_{\nu}}{2 n}, \frac{t+\xi_{\nu+1}}{2 n}\right), \quad \text { with } \nu \in[0, m]
$$

Then

$$
\left\{\frac{\alpha(2 n x+s+i)+\beta}{2 n}\right\} \in\left[\frac{t+l_{i}(\nu)}{2 n}, \frac{t+l_{i}(\nu)+1}{2 n}\right), \quad i=1, \ldots, m .
$$


Proof. Using (17), we have

$$
\begin{aligned}
z_{i} & =\left\{\frac{\alpha(2 n x+s+i)+\beta}{2 n}\right\}=\left\{\frac{\alpha(2 n x+s)+\beta}{2 n}+\frac{\alpha i}{2 n}\right\} \\
& =\left\{\frac{t+\xi_{\nu}+\epsilon+[\alpha i]+\{\alpha i\}}{2 n}\right\}, \text { with } 0 \leq \epsilon<\xi_{\nu+1}-\xi_{\nu} .
\end{aligned}
$$

From (15), it follows that

$$
z_{i}=\left\{\frac{t+[\alpha i]+\xi_{\nu}+1-\xi_{f(i)}+\epsilon}{2 n}\right\} .
$$

It is evident that if $f(i)>\nu$, then

$$
\xi_{\nu}+\epsilon-\xi_{f(i)}<\xi_{\nu+1}-\xi_{f(i)} \leq 0 .
$$

Applying (16), we get (18) with $l_{i}(\nu)=[\alpha i]$. Now let $f(i) \leq \nu$; then

$$
0 \leq \xi_{\nu}+\epsilon-\xi_{f(i)}<\xi_{\nu+1}
$$

By (16), we obtain (18) with $l_{i}(\nu)=[\alpha i]+1$.

Let us examine the conditions for the line $(z, \alpha z+\beta)_{z \geq 0}$ to intersect the square $\left(2 n x+s, 2 n y^{\prime}+t^{\prime}\right)+[0,1)^{2}$ where $s, t^{\prime}, x, y^{\prime}$ are integers, $0 \leq x, y^{\prime}<$ $b^{2 n^{2}}, 0 \leq s<2 n, 0 \leq t^{\prime}<2 n-\sqrt{n}$ :

$$
[\alpha(2 n x+s)+\beta] \leq 2 n y^{\prime}+t^{\prime}<\alpha(2 n x+s+1)+\beta .
$$

Consider the pair $(y, t)$ such that

$$
\begin{gathered}
{[\alpha(2 n x+s)+\beta]=2 n y+t} \\
2 n y+t \leq \alpha(2 n x+s)+\beta<2 n y+t+1 .
\end{gathered}
$$

Hence

$$
y+\frac{t}{2 n} \leq \frac{\alpha(2 n x+s)+\beta}{2 n}<y+\frac{t+1}{2 n} .
$$

We get that (20) is valid for some integer $y$ if and only if

$$
\left\{\frac{\alpha(2 n x+s)+\beta}{2 n}\right\} \in\left[\frac{t}{2 n}, \frac{t+1}{2 n}\right) .
$$

Let $U_{n}(N)\left(U_{n}^{*}(N)\right)$ be the subset of all lattice points $\left(2 n x+s, 2 n y^{\prime}+t^{\prime}\right)$ such that the line $(z, \alpha z+\beta)_{z \geq 0}$ intersects the square $\left(2 n x+s, 2 n y^{\prime}+t^{\prime}\right)+[0,1)^{2}$ where $0 \leq x, y^{\prime}<N, 0 \leq s<2 n$, and $0 \leq t^{\prime}<2 n$ (respectively $0 \leq t^{\prime}<$ $2 n-\sqrt{n})$.

Using (16) and (19), we have

$$
(1+[\alpha]) 2 n N \leq \# U_{n}(N) \leq(2+[\alpha]) 2 n N .
$$

It is easy to see that

$$
\# U_{n}(N)=\# U_{n}^{*}(N)+O(N \sqrt{n}) .
$$


Fix $x$ and $s$. We get from (19) that a lower bound of $2 n y^{\prime}+t^{\prime}$ is equal to

$$
[\alpha(2 n x+s)+\beta]=2 n y_{0}+t_{0}
$$

for some $y_{0}, t_{0}$. Since $\alpha$ is irrational, for given $\beta$ there exists an $n(\beta)$ such that for any greater $n,\{\alpha n+\beta\}>0$.

Again from (19) we have that for $n>n(\beta)$ a upper bound of $2 n y^{\prime}+t^{\prime}$ is equal to

$$
[\alpha(2 n x+s+1)+\beta]=2 n y_{1}+t_{1}
$$

for some $y_{1}, t_{1}$. Let $n>\max (n(\beta), \alpha)$ and $t \in[0,2 n-\sqrt{n}]$ satisfy $(20)$. We have from $(20),(24)$, and (25) that

$$
y_{1}=y_{0}=y \text { and } t_{0}=t .
$$

To determine $t_{1}$, we use (20), (21), and (25):

$$
\left\{\frac{\alpha(2 n x+s)+\beta}{2 n}\right\} \in\left[\frac{t}{2 n}, \frac{t+1}{2 n}\right)
$$

and

$$
\left\{\frac{\alpha(2 n x+s+1)+\beta}{2 n}\right\} \in\left[\frac{t_{1}}{2 n}, \frac{t_{1}+1}{2 n}\right) .
$$

Clearly, there exists a unique $\nu_{0}=\nu_{0}(x, s, t) \in[0, m]$ such that

$$
\left\{\frac{\alpha(2 n x+s)+\beta}{2 n}\right\} \in\left[\frac{t+\xi_{\nu_{0}}}{2 n}, \frac{t+\xi_{\nu_{0}+1}}{2 n}\right) .
$$

Applying Lemma 1 with $i=1$ we get that

$$
t_{1}=t+l_{1}\left(\nu_{0}\right)
$$

From (19), (24)-(26), and (28), we get that $U_{n}^{*}(N)$ decomposes as follows.

$$
U_{n}^{*}(N)=\bigcup_{0 \leq x<N} \bigcup_{0 \leq s<2 n} B(x, s)
$$

where

$$
B(x, s)=\bigcup_{0 \leq t<2 n-\sqrt{n} 0 \leq j \leq l_{1}\left(\nu_{0}\right)}\left\{z(x, s, j) \mid \alpha_{x, s} \in \Delta_{t, \nu_{0}}\right\}
$$

and

$$
\begin{gathered}
\alpha_{x, s}=\left\{\frac{\alpha(2 n x+s)+\beta}{2 n}\right\}, \quad \Delta_{t, \nu}=\left[\frac{t+\xi_{\nu}}{2 n}, \frac{t+\xi_{\nu+1}}{2 n}\right), \\
z(x, s, j)=(2 n x+s,[\alpha(2 n x+s)+\beta]+j) .
\end{gathered}
$$

Now, it follows from (31) that for given $x$ and $s$ there is a unique pair $(t, \nu)$ with $t \in[0,2 n), \nu \in[0, m]$ such that

$$
\alpha_{x, s} \in \Delta_{t, \nu}
$$


By (30), we obtain that

$$
B(x, s)=\bigcup_{0 \leq t<2 n-\sqrt{n}} \bigcup_{0 \leq \nu \leq m} \bigcup_{0 \leq j \leq l_{1}(\nu)}\left\{z(x, s, j) \mid \alpha_{x, s} \in \Delta_{t, \nu}\right\} .
$$

Using (29), we get

(33)

$$
U_{n}^{*}(N)=\bigcup_{0 \leq x<N} \bigcup_{0 \leq s<2 n} \bigcup_{0 \leq t<2 n-\sqrt{n}} \bigcup_{0 \leq \nu \leq m} \bigcup_{0 \leq j \leq l_{1}(\nu)}\left\{z(x, s, j) \mid \alpha_{x, s} \in \Delta_{t, \nu}\right\}
$$

or

$$
U_{n}^{*}(N)=\bigcup_{0 \leq s<2 n} \bigcup_{0 \leq t<2 n-\sqrt{n}} \bigcup_{0 \leq \nu \leq m} \bigcup_{0 \leq j \leq l_{1}(\nu)}\left\{z(x, s, j) \mid x \in E_{\nu s}(t, N)\right\},
$$

where

$$
E_{\nu s}(t, N)=\left\{x \in[0, N) \mid \alpha_{x, s} \in \Delta_{t, \nu}\right\} .
$$

We consider the case $\alpha<1$ (hence $x \geq y$ ) and $s \geq t$ first. We examine the interval $s \in[\sqrt{n}, 2 n-\sqrt{n}]$. For $s \in[0, \sqrt{n})$ and $s \in[2 n-\sqrt{n}, 2 n)$, we use a trivial estimate.

Let

$$
U_{n}^{\prime}(N)=\bigcup_{0 \leq t \leq s<2 n-\sqrt{n}} \bigcup_{0 \leq \nu \leq m} \bigcup_{0 \leq j \leq l_{1}(\nu)}\left\{z(x, s, j) \mid x \in E_{\nu s}(t, N)\right\}
$$

and

$$
E_{\nu s}\left(t, Q_{1}, Q_{2}\right)=\left\{x \in\left[Q_{1}, Q_{1}+Q_{2}\right) \mid \alpha_{x, s} \in \Delta_{t, \nu}\right\} .
$$

For $z \in U_{n}(N)$ denote by $z^{(1)}, \ldots, z^{(m)}$ the sequence of $z^{(i)} \in U_{n}(N),(i=$ $1, \ldots, m)$ stretching in succession, such that $z^{(1)}=z$. For $z=z(x, s, j)=$ $(2 n x+s,[\alpha(2 n x+s)+\beta]+j)$ with $j \in\left[0, l_{1}(\nu)\right]$, denote by $z^{(i)}(x, s, j)$ a vector $z^{(i)}$ :

$$
z^{(i)}(x, s, j)=z^{(i)}, \quad i=1, \ldots, m .
$$

Let $G_{m}=\left(g_{1}, \ldots, g_{m}\right)$ be a block of digits i.e., $g_{i} \in \Delta_{b}, i=1, \ldots, m$,

$$
V_{n}\left(N, G_{m}\right)=\left\{z \in U_{n}(N) \mid G_{m}=\left(\omega_{n}\left(z^{(1)}\right), \ldots, \omega_{n}\left(z^{(m)}\right)\right)\right\}
$$

is the set of the linear indices of beginning of a block $G_{m}$ in the linear configuration of digits, confined to the $\omega_{n}$-square configuration.

Define also

$$
V_{n}^{*}\left(N, G_{m}\right)=\left\{z \in U_{n}^{*}(N) \mid G_{m}=\left(\omega_{n}\left(z^{(1)}\right), \ldots, \omega_{n}\left(z^{(m)}\right)\right)\right\} .
$$

Let

$$
\chi_{\omega_{n} G_{m}}(z(x, s, j))= \begin{cases}1 & \text { if } z^{(i)}(x, s, j)=g_{i}, \quad i=1, \ldots, m \\ 0 & \text { otherwise }\end{cases}
$$


Applying (33) and (39)-(41), we obtain that

$$
\begin{array}{r}
V_{n}^{*}\left(N, G_{m}\right)=\bigcup_{0 \leq x<N} \bigcup_{0 \leq s<2 n} \bigcup_{0 \leq t<2 n-\sqrt{n}} \bigcup_{0 \leq \nu \leq m} \bigcup_{0 \leq j \leq l_{1}(\nu)}\{z(x, s, j) \\
\left.\mid \chi_{\omega_{n} G_{m}}(z(x, s, j))=1, \quad \alpha_{x, s} \in \Delta_{t, \nu}\right\} .
\end{array}
$$

Analogously to (34) and (35), we have that

$$
V_{n}^{*}\left(N, G_{m}\right)=\bigcup_{0 \leq s<2 n} \bigcup_{0 \leq t<2 n-\sqrt{n}} \bigcup_{0 \leq \nu \leq m} \bigcup_{0 \leq j \leq l_{1}(\nu)}\left\{z(x, s, j) \mid x \in A_{j \nu s}(t, N)\right\},
$$

where

(43) $A_{j \nu s}(t, N)=\left\{x \in[0, N) \mid \alpha_{x, s} \in \Delta_{t, \nu}\right.$, and $\left.\chi_{\omega_{n} G_{m}}(z(x, s, j))=1\right\}$.

Let

$$
\begin{gathered}
V_{n}^{\prime}\left(N, G_{m}\right)=\bigcup_{0 \leq t \leq s<2 n-\sqrt{n}} \bigcup_{0 \leq \nu \leq m} \bigcup_{0 \leq j \leq l_{1}(\nu)}\{(2 n x+s,[\alpha(2 n x+s)+\beta]+j) \\
\left.(44) \quad \mid x \in A_{j \nu s}(t, N)\right\},
\end{gathered}
$$

and

$$
\begin{array}{ll}
A_{j \nu s}\left(t, Q_{1}, Q_{2}\right)=\left\{x \in\left[Q_{1}, Q_{1}+Q_{2}\right) \mid\right. & \alpha_{x, s} \in \Delta_{t, \nu} \\
& \text { and } \left.\chi_{\omega_{n} G_{m}}(z(x, s, j))=1\right\} .
\end{array}
$$

Next we write an analytic expression for the sequence $\left(z^{(i)}(x, s, j)\right)_{1 \leq i \leq m}$ : For given integers $t \in[0,2 n-\sqrt{n}), x, s, \nu$, and $j \in\left[0, l_{1}(\nu)\right)$ put

$$
\alpha_{x, s} \in \Delta_{t, \nu} .
$$

Using (19)-(21) and Lemma 1 we have similarly to (24)-(28) that the part of $U_{n}^{\prime}(N)$ corresponding to the interval $\left[2 n x+s+i^{\prime}, 2 n x+s+i^{\prime}+1\right)$ (with condition (46)) is equal to

$$
\left(\left(2 n x+s+i^{\prime},[\alpha(2 n x+s)+\beta]+l_{i^{\prime}}(\nu)+v\right)\right)
$$

where $v=j \delta_{i^{\prime}}, j \delta_{i^{\prime}}+1, \ldots, l_{i^{\prime}+1}(\nu)-l_{i^{\prime}}(\nu)-1, \quad i^{\prime}=0,1, \ldots, m-1$, with $l_{0}(\nu)=0$,

$$
\delta_{i}^{\prime}= \begin{cases}1 & \text { if } i^{\prime}=0 \\ 0 & \text { otherwise }\end{cases}
$$

Hence, the sequence $\left(z^{(i)}(x, s, j)\right)_{1 \leq i \leq m}(38)$ coincides with the first $m$ terms of the following sequence

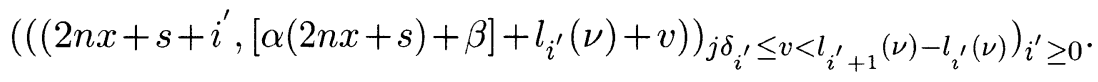


Now let $\left(k_{n}\right)_{n \geq 1}$ be an increasing sequence of integers from the set

$$
\begin{gathered}
\left\{\left(s+i^{\prime}\right)^{2}+t+l_{i^{\prime}}(\nu)+v+1 \mid v=j \delta_{i^{\prime}}, j \delta_{i^{\prime}}+1, \ldots, l_{i^{\prime}+1}(\nu)-l_{i^{\prime}}(\nu)-1,\right. \\
\left.i^{\prime}=0,1, \ldots, m-1\right\} .
\end{gathered}
$$

Observe that the equality

$$
\chi_{\omega_{n} G_{m}}(z(x, s, j))=1
$$

(with condition (46) or (21)) is equivalent to the assertion (found in (5), (6), (48), and (49)) that the $\left(k_{i}-1\right)$ th digit of the $b$-expansion of $x^{2}+y$ is equal to $g_{i}(i=1, \ldots, m)$ :

$$
x^{2}+y=x_{1}+g_{1} b^{k_{1}-1}+x_{2} b^{k_{1}}+g_{2} b^{k_{2}-1}+\ldots+x_{m} b^{k_{m-1}}+g_{m} b^{k_{m}-1}+x_{m+1} b^{k_{m}},
$$

where $x_{i} \in\left[0, b^{k_{i}-k_{i-1}-1}\right)$ are integers $(i=1, \ldots, m), k_{0}=0$, and $y=y(x, s)$ is a function of $(x, s)$ (see (20)).

In the sequel we consider $y$ as $y=y(x, s)$. Using (43), (45), and (50), we have that

$$
\begin{gathered}
A_{j \nu s}(t, N)=\left\{x \in[0, N) \mid \alpha_{x, s} \in \Delta_{t, \nu}, \text { and } x^{2}+y=x_{1}\right. \\
\left.+g_{1} b^{k_{1}-1}+x_{2} b^{k_{1}}+g_{2} b^{k_{2}-1}+\ldots+x_{m} b^{k_{m-1}}+g_{m} b^{k_{m}-1}+x_{m+1} b^{k_{m}}\right\}
\end{gathered}
$$

and

$$
\begin{gathered}
A_{j \nu s}\left(t, Q_{1}, Q_{2}\right)=\left\{x \in\left[Q_{1}, Q_{1}+Q_{2}\right) \mid \alpha_{x, s} \in \Delta_{t, \nu}, \text { and } x^{2}+y=x_{1}\right. \\
\left.+g_{1} b^{k_{1}-1}+x_{2} b^{k_{1}}+g_{2} b^{k_{2}-1}+\ldots+x_{m} b^{k_{m-1}}+g_{m} b^{k_{m}-1}+x_{m+1} b^{k_{m}}\right\}
\end{gathered}
$$

Let

$$
B_{N}(s, t, j, \nu, g)=\#\left\{x \in[0, N) \mid \alpha_{x s} \in \Delta_{t \nu},\right. \text { and }
$$

$$
\left.\left\{\left(x^{2}+y\right) b^{-k_{m}}\right\} \in\left[\frac{g}{b^{k_{m}-k_{1}+1}}, \frac{g+1}{b^{k_{m}-k_{1}+1}}\right)\right\} .
$$

Lemma 2. Let $N \in\left[b^{2 n^{2}-5 n}, b^{2 n^{2}}\right), s \in[\sqrt{n}, 2 n-\sqrt{n}]$. Then

$$
\# A_{j \nu s}(t, N)=\sum_{x_{2}=0}^{b^{k_{2}-k_{1}-1}-1} \ldots \sum_{x_{m}=0}^{b^{k_{m}-k_{m-1}-1}-1} B_{N}(s, t, j, \nu, g)
$$

where

$$
g=g\left(x_{2}, . ., x_{m}\right)=g_{1}+x_{2} b+g_{2} b^{k_{2}-k_{1}}+\ldots+x_{m} b^{k_{m-1}-k_{1}+1}+g_{m} b^{k_{m}-k_{1}} \text {. }
$$


Proof. Using (51) and (55) we get

$$
\begin{array}{r}
A_{j \nu s}(t, N)=\bigcup_{x_{2}=0}^{b^{k_{2}-k_{1}-1}-1} \ldots \bigcup_{x_{m}=0}^{b^{k_{m}-k_{m-1}-1}-1}\left\{x \in[0, N) \mid \alpha_{x, s} \in \Delta_{t \nu}\right. \text {, and } \\
\left.x^{2}+y=x_{1}+g b^{k_{1}-1}+x_{m+1} b^{k_{m}}\right\} .
\end{array}
$$

Bearing in mind that the condition

$$
x^{2}+y=x_{1}+g b^{k_{1}-1}+x_{m+1} b^{k_{m}}
$$

is equivalent to the condition

$$
\left\{\left(x^{2}+y\right) b^{-k_{m}}\right\} \in\left[\frac{g}{b^{k_{m}-k_{1}+1}}, \frac{g+1}{b^{k_{m}-k_{1}+1}}\right)
$$

with any integer $x_{1} \in\left[0, b^{k_{1}-1}\right), \quad x_{m+1} \geq 0$ (integer $g \in\left[0, b^{k_{m}-k_{1}+1}\right.$ ) is fixed) we obtain from (53) the assertion of the lemma.

Let $q_{1}<q_{2} \ldots<q_{n}<\ldots$ be a sequence of denominators of the continued fraction of $\alpha$,

$$
\alpha=\frac{p_{r}}{q_{r}}+\frac{\theta_{r}}{q_{r} q_{r+1}}, \text { with } \quad\left(p_{r}, q_{r}\right)=1, \quad\left|\theta_{r}\right| \leq 1
$$

and

$$
q_{r} \leq b^{(s+6 m)^{2} / 2}<q_{r+1}
$$

$(r=r(s, m)$ is the function of $s, m$ with fixed integer $m$, and $s=1,2, \ldots)$.

We will consider two cases. In the first case $q_{r}$ is large $\left(q_{r} \geq n^{6} b^{2 s m}\right.$, the growth of $q_{r}$ is small, case of almost all $\alpha$ ). In the second case $q_{r}$ is small ( $q_{r} \leq n^{6} b^{2 s m}$, the growth of $q_{r}$ is large), case of almost no $\alpha$ (the complicated case).

\subsection{1. case $q_{r}$ large.}

In this subsection, we prove that the frequency of the appearance of the block of digits $G_{m}$ in the sequence $\left(u_{\alpha, \beta}(n)\right)_{n=1}^{N}$ tends to $b^{-m}$. According to (42) and (51)-(54), it is sufficient to get the estimate of $B_{N}(s, t, j, \nu, g)$ (see (53)) to prove the above statement. We use the estimate of exponential sums (Lemma 3 ) and the Erdös-Turan-Koksma inequality (13) to compute $B_{N}(s, t, j, \nu, g)$.

Lemma 3. Let $q_{r} \geq n^{6} b^{2 s m}, \quad N \in\left[b^{2 n^{2}-5 n}, b^{2 n^{2}}\right), s \in[\sqrt{n}, 2 n-\sqrt{n}]$, $0<\max \left(\left|m_{1}\right|,\left|m_{2}\right|\right) \leq M=n^{4} b^{2 s m}$,

$$
S_{N}\left(m_{1}, m_{2}\right)=\sum_{x=0}^{N-1} e\left(m_{1}\left(x^{2}+\alpha x+\gamma\right) b^{-k_{m}}+m_{2}(\alpha(2 n x+s)+\beta) / 2 n\right) .
$$

Then

$$
\left|S_{N}\left(0, m_{2}\right)\right| \leq q_{r}
$$


$(60)$

$$
\left|S_{N}\left(m_{1}, m_{2}\right)\right|=O\left(N n^{-6} b^{-2 s m}\right), \quad m_{1} \neq 0 .
$$

Proof. Let $m_{1}=0$. It is easy to see that

$$
\sigma_{1}=\left|S_{N}\left(0, m_{2}\right)\right|=\left|\sum_{x=0}^{N-1} e\left(m_{2} \alpha x\right)\right| .
$$

Using (14) we have

$$
\sigma_{1} \leq \min \left(N, \frac{1}{2\left\|m_{2} \alpha\right\|}\right) .
$$

Bearing in mind (56) and the condition of Lemma 3, we obtain that

$$
0<\left|m_{2}\right| \leq n^{4} b^{2 s m}<q_{r} / 2
$$

and

$$
\left\|m_{2} \alpha\right\|=\left\|m_{2}\left(\frac{p_{r}}{q_{r}}+\frac{\theta_{r}}{q_{r} q_{r+1}}\right)\right\| \geq\left|\frac{1}{q_{r}}-\frac{1}{2 q_{r+1}}\right| \geq \frac{1}{2 q_{r}} .
$$

We have from (62) that $\sigma_{1} \leq q_{r}$. Hence (59) follows from (61). Assume $m_{1} \neq 0$. It is easy to see that

$$
\begin{aligned}
\sigma_{2}^{2} & =\left|S_{N}\left(m_{1}, m_{2}\right)\right|^{2} \\
& =\sum_{x_{1}, x_{2}=0}^{N-1} e\left(m_{1}\left(x_{1}^{2}-x_{2}^{2}+\alpha\left(x_{1}-x_{2}\right)\right) b^{-k_{m}}+m_{2} \alpha\left(x_{1}-x_{2}\right)\right) .
\end{aligned}
$$

Let $x_{1}-x_{2}=u$. Then $u \in[-N+1, N-1], x_{2}=x_{1}-u \in[0, N-1], x_{1}+x_{2}=$ $2 x_{1}-u, x_{1}^{2}-x_{2}^{2}=u\left(2 x_{1}-u\right), x_{1} \in[\max (0, u), \min (N-1, N-1+u)]$. Hence

$$
\begin{aligned}
\sigma_{2}^{2} & =\sum_{u=-N+1}^{N-1} \sum_{x_{1}=\max (0, u)}^{\min (N-1, N-1+u)} e\left(m_{1}\left(2 u x_{1}-u^{2}+\alpha u\right) b^{-k_{m}}+m_{2} \alpha u\right) \\
& \leq \sum_{u=-N+1}^{N-1}\left|\sum_{x_{1}=\max (0, u)}^{\min (N-1, N-1+u) \mid} e\left(2 m_{1} u x_{1} b^{-k_{m}}\right)\right| .
\end{aligned}
$$

By (14) we get

$$
\sigma_{2}^{2} \leq \sum_{u=-N+1}^{N-1} \min \left(2 N, \frac{1}{2\left\|2 m_{1} u b^{-k_{m}}\right\|}\right) .
$$


Let $\left(2 m_{1}, b^{k_{m}}\right)=f, u=u_{1}+u_{2} b^{k_{m}} / f$ with $u_{1} \in\left[0, b^{k_{m}} / f-1\right]$ and $u_{2} \in$ $\left[-N_{1}, N_{1}\right]$, where $N_{1}=\left[f(N-1) b^{-k_{m}}\right]+1$. It is easy to see that

$$
\begin{aligned}
\sigma_{2}^{2} & \leq \sum_{u_{2}=-N_{1}}^{N_{1}}\left(2 N+\sum_{u_{1}=1}^{b^{k_{m}} / f-1} \frac{1}{2\left\|f u_{1} b^{-k_{m}}\right\|}\right) \\
& \leq\left(\frac{2 N}{b^{k_{m}} / f}+3\right)\left(2 N+\left(b^{k_{m}} / f\right) \log b^{k_{m}}\right) \\
& \leq 4 N^{2}\left|m_{1}\right| b^{-k_{m}}+6 N+2 N k_{m} \log b+3 b^{k_{m}} k_{m} \log b .
\end{aligned}
$$

Hence

$$
\sigma_{2}=O\left(N b^{-k_{m} / 2} \sqrt{\left|m_{1}\right|}+{\sqrt{k_{m}}}_{b^{k_{m} / 2}}\right) .
$$

Using (49) we obtain that $s^{2}+1 \leq k_{m} \leq(s+m)^{2}+\alpha m+2$ and

$$
\sigma_{2}=O\left(N b^{-s^{2} / 2} n^{2} b^{s m}+(s+m) b^{(s+m)^{2} / 2}\right) .
$$

Taking into account that $s \in[\sqrt{n}, 2 n-\sqrt{n}]$, we get

$$
N n^{2} b^{s m-s^{2} / 2}=O\left(N n^{-6} b^{-2 s m}\right),
$$

and

$$
\begin{aligned}
(s+m)^{2} b^{(s+m)^{2} / 2} & =O\left(n^{2} b^{(s+4 m)^{2} / 2-2 s m}\right)=O\left(n^{2} b^{(2 n-\sqrt{n}+4 m)^{2} / 2-2 s m}\right) \\
& =o\left(n^{-6} b^{2 n^{2}-5 n-2 s m}\right)=O\left(N n^{-6} b^{-2 s m}\right) .
\end{aligned}
$$

Using (63), and (66)-(68), we obtain (60).

Lemma 4. Let $q_{r} \geq n^{6} b^{2 s m}, \quad N \in\left[b^{2 n^{2}-5 n}, b^{2 n^{2}}\right), \quad s \in[\sqrt{n}, 2 n-\sqrt{n}]$. Then

$$
\begin{gathered}
N D_{N}=N D_{N}\left(\left(\left\{\left(x^{2}+\alpha x+\gamma\right) b^{-k_{m}}\right\},\{(\alpha(2 n x+s)+\beta) / 2 n\}\right)_{x=0}^{N-1}\right) \\
=O\left(N n^{-4} b^{-k_{m}+k_{1}}\right) .
\end{gathered}
$$

Proof. Using the Erdös-Turan-Koksma inequality (13) with $k=2, M=$ $n^{4} b^{2 s m}$, and (58), we obtain that

$$
N D_{N}=O\left(N n^{-4} b^{-2 s m}+\sigma_{3}+\sigma_{4}\right)
$$

where

$$
\sigma_{3}=\sum_{0<\left|m_{2}\right| \leq M} \frac{\left|S\left(0, m_{2}\right)\right|}{\bar{m}_{2}}
$$

and

$$
\sigma_{4}=\sum_{0<\max \left(\left|m_{1}\right|,\left|m_{2}\right|\right) \leq M, m_{1} \neq 0} \frac{\left|S\left(m_{1}, m_{2}\right)\right|}{\bar{m}_{1} \bar{m}_{2}}
$$


Applying (59) we have that

$$
\sigma_{3} \leq 2 \sum_{m_{2}=1}^{M} \frac{q_{r}}{\bar{m}_{2}} \leq 2 q_{r}(1+\log M) .
$$

Using (57) and the conditions of Lemma 4, we obtain that

$$
\begin{aligned}
\sigma_{3} & =O\left(b^{(s+6 m)^{2} / 2} s m\right)=O\left(n b^{(2 n-\sqrt{n}+6 m)^{2} / 2}\right) \\
& =O\left(n^{-4} b^{2 n^{2}-5 n-4 n m}\right)=O\left(n^{-4} b^{2 n^{2}-5 n-2 s m}\right)=O\left(n^{-4} N b^{-2 s m}\right) .
\end{aligned}
$$

From (60) and (71), we get

$$
\begin{gathered}
\sigma_{4}=O\left(n^{-6} N b^{-2 s m} \sum_{\left|m_{1}\right|,\left|m_{2}\right| \leq M} \frac{1}{\bar{m}_{1} \bar{m}_{2}}\right) \\
(73)=O\left(n^{-6} N b^{-2 s m}(\log M)^{2}\right)=O\left(s^{2} n^{-6} N b^{-2 s m}\right)=O\left(n^{-4} N b^{-2 s m}\right) .
\end{gathered}
$$

Combining (73) with (69)-(72), we have that

$$
N D_{N}=O\left(n^{-4} N b^{-2 s m}\right) \text {. }
$$

By (49), and (16) we obtain

$$
-k_{m}+k_{1} \geq s^{2}-(s+m)^{2}-\alpha m-1=-2 s m-m^{2}-\alpha m-1 .
$$

The assertion of the lemma follows from (74) .

Let

$$
B_{N}\left(s, t, \nu, \gamma, \gamma_{1}, \gamma_{2}\right)
$$

$$
=\#\left\{x \in[0, N) \mid \alpha_{x s} \in \Delta_{t \nu}, \quad\left\{\left(x^{2}+\alpha x+\gamma\right) b^{-k_{m}}\right\} \in\left[\gamma_{1}, \gamma_{2}\right)\right\} .
$$

Corollary 1. Let $0 \leq \gamma_{1}<\gamma_{2} \leq 1$. Under the assumptions of Lemma 4 ,

$$
B_{N}\left(s, t, \nu, \gamma, \gamma_{1}, \gamma_{2}\right)=N \frac{\xi_{\nu+1}-\xi_{\nu}}{2 n}\left(\gamma_{2}-\gamma_{1}\right)+O\left(N n^{-4} b^{-k_{m}+k_{1}}\right) .
$$

Proof. The proof follows from (10), (31), and Lemma 4.

Apply (20) to get a formula for the number $x^{2}+y($ where $y=y(x, s))$ :

$$
[\alpha(2 n x+s)+\beta]=2 n y+t \text {. }
$$

Let also

$$
\begin{aligned}
& \alpha_{x, s}=\left\{\frac{\alpha(2 n x+s)+\beta}{2 n}\right\} \in \Delta_{t, \nu}=\left[\frac{t+\xi_{\nu}}{2 n}, \frac{t+\xi_{\nu+1}}{2 n}\right), \\
& \alpha_{x, s}=\frac{t+\epsilon}{2 n}, \text { where } \epsilon \in[0,1) .
\end{aligned}
$$

Then

for an integer $i$. Hence

$$
\frac{\alpha(2 n x+s)+\beta}{2 n}=i+\frac{t+\epsilon}{2 n}
$$

$$
\{\alpha(2 n x+s)+\beta\}=\{2 n i+t+\epsilon\}=\epsilon .
$$


Using (77) we obtain

$$
\begin{aligned}
y=\frac{[\alpha(2 n x+s)+\beta]-t}{2 n} & =\frac{\alpha(2 n x+s)+\beta-t-\{\alpha(2 n x+s)+\beta\}}{2 n} \\
& =\frac{\alpha(2 n x+s)+\beta-t}{2 n}-\frac{\epsilon}{2 n} .
\end{aligned}
$$

Therefore

$$
x^{2}+y=x^{2}+\alpha x+\frac{\alpha s+\beta-t}{2 n}-\frac{\epsilon}{2 n} .
$$

Lemma 5. Let $0 \leq g<b^{k_{m}-k_{1}+1}$. Under the assumptions of Lemma 4,

$$
B_{N}(s, t, j, \nu, g)=N \frac{\xi_{\nu+1}-\xi_{\nu}}{2 n} b^{k_{1}-k_{m}-1}\left(1+O\left(n^{-3}\right)\right),
$$

where $B_{N}(s, t, j, \nu, g)$ is defined in (53).

Proof. We have from (78) that if

$$
\left\{\left(x^{2}+\alpha x+\gamma_{0}\right) b^{-k_{m}}\right\} \in\left[\frac{g}{b^{k_{m}-k_{1}+1}}+\theta_{0}, \frac{g+1}{b^{k_{m}-k_{1}+1}}\right)
$$

with $\theta_{0}=\frac{1}{2 n b^{k_{m}}}$, and $\gamma_{0}=\frac{\alpha s+\beta-t}{2 n}$, then

$$
\left\{\left(x^{2}+y\right) b^{-k_{m}}\right\} \in\left[\frac{g}{b^{k_{m}-k_{1}+1}}, \frac{g+1}{b^{k_{m}-k_{1}+1}}\right) .
$$

Hence and from (53) and (76) we get

$$
B_{N}\left(s, t, \nu, \gamma_{0}, \frac{g}{b^{k_{m}-k_{1}+1}}+\theta_{0}, \frac{g+1}{b^{k_{m}-k_{1}+1}}\right) \leq B_{N}(s, t, j, \nu, g) .
$$

Conversely, assume (80), then

$$
\left\{\left(x^{2}+\alpha x+\gamma_{0}\right) b^{-k_{m}}\right\} \in\left[\frac{g}{b^{k_{m}-k_{1}+1}}-\theta_{0}, \frac{g+1}{b^{k_{m}-k_{1}+1}}\right) \text { for } g \geq 1
$$

and

$$
\left\{\left(x^{2}+\alpha x+\gamma_{0}\right) b^{-k_{m}}\right\} \in\left[0, \frac{1}{b^{k_{m}-k_{1}+1}}\right) \cup\left[1-\theta_{0}, 1\right) \text { for } g=0 .
$$

Hence

$$
\begin{aligned}
B_{N}(s, t, j, \nu, g) \leq & B_{N}\left(s, t, \nu, \gamma_{0}, \max \left(0, \frac{g}{b^{k_{m}-k_{1}+1}}-\theta_{0}\right), \frac{g+1}{b^{k_{m}-k_{1}+1}}\right) \\
& +B_{N}\left(s, t, \nu, \gamma_{0}, 1-\theta_{0}, 1\right) .
\end{aligned}
$$

Using Corollary 1, we have

(83) $B_{N}(s, t, j, \nu, g)=N \frac{\xi_{\nu+1}-\xi_{\nu}}{2 n}\left(\frac{1}{b^{k_{m}-k_{1}+1}}+\epsilon_{1} \theta_{0}\right)+O\left(N n^{-4} b^{-k_{m}+k_{1}}\right)$, with $\left|\epsilon_{1}\right|<2$. Applying (49) and (80), we get that

$$
b^{k_{1}-k_{m}-1}+\epsilon \theta_{0}=b^{k_{1}-k_{m}-1}\left(1+O\left(n^{-1} b^{-k_{1}}\right)\right)=b^{k_{1}-k_{m}-1}\left(1+O\left(n^{-3}\right)\right) \text {. }
$$


Bearing in mind (83) and that $\xi_{\nu+1}-\xi_{\nu} \geq c>0(\nu=0,1, \ldots, m)$, we obtain (79).

Corollary 2. Let $0 \leq t \leq s, j \in\left[0, l_{1}(\nu)\right]$. Under the assumptions of Lemma 4,

$$
\# A_{j \nu s}(t, N)=b^{-m} N \frac{\xi_{\nu+1}-\xi_{\nu}}{2 n}\left(1+O\left(1 / n^{3}\right)\right) .
$$

The proof follows from Lemma 2 and Lemma 5.

Lemma 6. Let $0 \leq t \leq s$. Under the assumptions of Lemma 4 ,

$$
\# A_{j \nu s}(t, N)=b^{-m} \# E_{\nu s}(t, N)(1+O(1 / n)) \text {. }
$$

Proof. We get from (31), (37), and (56) that

$E_{\nu s}\left(t, Q_{1}, q_{r}\right)=\left\{x \in\left[0, q_{r}\right) \mid\left\{\frac{x p_{r}}{q_{r}}+\frac{\left.\alpha\left(2 n Q_{1}+s\right)+\beta\right)}{2 n}+\frac{\theta_{r} x}{q_{r} q_{r+1}}\right\} \in \Delta_{t, \nu}\right\}$.

Using (57) and that $\left(p_{r}, q_{r}\right)=1$, we obtain

$$
E_{\nu s}\left(t, Q_{1}, q_{r}\right)=\left\{x \in\left[0, q_{r}\right) \mid\left\{\frac{x}{q_{r}}+\gamma+\frac{\theta(x)}{q_{r}}\right\} \in \Delta_{t, \nu}\right\}
$$

with $\gamma=\frac{\alpha\left(2 n Q_{1}+s\right)+\beta}{2 n},|\theta(x)| \leq 1$.

Recalling the definition (31) of $\Delta_{t \nu}$, get

$$
\# E_{\nu s}\left(t, Q_{1}, q_{r}\right)=q_{r}\left(\frac{\xi_{\nu+1}-\xi_{\nu}}{2 n}\right)+4 \epsilon, \quad \text { where }|\epsilon| \leq 1
$$

Taking into account that $\xi_{\nu+1}-\xi_{\nu} \geq c>0 \quad(\nu=0,1, \ldots, m)$ and the condition of the lemma, we obtain

$$
\# E_{\nu s}\left(t, Q_{1}, q_{r}\right)=q_{r}\left(\frac{\xi_{\nu+1}-\xi_{\nu}}{2 n}\right)(1+O(1 / n))
$$

From (35), (37), and the condition of the lemma, we get

$$
\begin{aligned}
\# E_{\nu s}(t, N) & =\sum_{0 \leq i<\left[N / q_{r}\right]} \# E_{\nu s}\left(t, i q_{r}, q_{r}\right)+\epsilon q_{r} \\
& =\left[N / q_{r}\right] q_{r}\left(\frac{\xi_{\nu+1}-\xi_{\nu}}{2 n}\right)(1+O(1 / n))+\epsilon q_{r} \\
& =N\left(\frac{\xi_{\nu+1}-\xi_{\nu}}{2 n}\right)(1+O(1 / n)) .
\end{aligned}
$$

Applying Corollary 2, the assertion of Lemma 6 follows.

\subsubsection{Small $q_{r}$ (the complicated case).}

The main idea of this subsection is the partition of the interval of summation into $q_{r}$ arithmetical progressions $\left(x+v q_{r}\right)_{v \geq 0}$ with $x \in\left[0, q_{r}\right)$. Next we fix the integer $x$ and compute (similarly to Lemma 3 - Lemma 5) the number of occurrences of the block of digits $G_{m}$ in the sequence $\left(u_{\alpha, \beta}(n)\right)_{n=1}^{N}$. 
We obtain in Lemma 10 the main estimate of this subsection for the special Diophantine condition. We find in Lemma 11 the set of parameters, for which the above Diophantine condition is true. In Lemma 12 we partition the interval of summation (according to Lemma 11) to essential intervals, and to auxiliary intervals. We use the estimate of Lemma 10 for the essential intervals and the trivial estimate for auxiliary intervals. We collect all the gotten results in Lemma 13 - Lemma 15.

Lemma 7. Let $q_{r} \leq n^{6} b^{2 s m}, \quad P \geq n^{4} b^{s^{2} / 2+3 s m}, s \in[\sqrt{n}, 2 n-\sqrt{n}]$, $0<|m| \leq M=n^{4} b^{2 s m}, x \in\left[H,(H+1) q_{r}\right), H \geq 0$,

$$
\left.S_{P}(m)=\sum_{v=0}^{P-1} e\left(m\left(\left(x+v q_{r}\right)^{2}+\alpha\left(x+v q_{r}\right)+\gamma\right) b^{-k_{m}}\right)\right) .
$$

Then

$$
\left|S_{P}(m)\right|=O\left(P n^{-3} b^{-2 s m}\right) .
$$

Proof. Similarly to (63)-(64), we have that

$$
\left|S_{P}(m)\right|^{2} \leq \sum_{u=-P+1}^{P-1} \min \left(2 P, \frac{1}{2\left\|2 m q_{r}^{2} b^{-k_{m}} u\right\|}\right) .
$$

Let $\left(2 m q_{r}^{2}, b^{k_{m}}\right)=f$. We note that

$$
1 \leq f \leq 2 m q_{r}^{2}=O\left(n^{16} b^{6 s m}\right) .
$$

By (49) and by the conditions of the lemma, we get similarly to (65) that

$$
\begin{aligned}
\left|S_{P}(m)\right|^{2} & =O\left(P^{2} f b^{-k_{m}}+P k_{m}+b^{k_{m}} k_{m}\right) \\
& =O\left(P^{2} n^{16} b^{6 s m-s^{2}}+s^{2} P+s^{2} b^{(s+m)^{2}}\right) \\
& =O\left(P^{2} b^{-10 s m}+s^{2} n^{-8} P^{2} b^{(s+m)^{2}-s^{2}-6 s m}\right) \\
& =O\left(n^{-6} P^{2} b^{-4 s m}\right) .
\end{aligned}
$$

Lemma 8. Let $q_{r} \leq n^{6} b^{2 s m}, \quad P \geq n^{4} b^{s^{2} / 2+3 s m}, \quad s \in[\sqrt{n}, 2 n-\sqrt{n}]$, $x \in\left[H,(H+1) q_{r}\right), \bar{H} \geq 0$. Then

$$
P D_{P}=P D_{P}\left(\left(\left\{\left(\left(x+v q_{r}\right)^{2}+\alpha\left(x+v q_{r}\right)+\gamma\right) b^{-k_{m}}\right\}\right)_{v=0}^{P-1}\right)=O\left(P n^{-4} b^{-k_{m}+k_{1}}\right) \text {. }
$$

Proof. Using (13) with $k=1, M=n^{4} b^{2 s m}$, Lemma 7, and (85), we obtain that

$$
\begin{aligned}
P D_{P}= & O\left(P n^{-4} b^{-2 s m}+\sum_{0<|m| \leq M} \frac{\left|S_{P}(m)\right|}{\bar{m}}\right)=O\left(P n^{-4} b^{-2 s m}\right. \\
& \left.+P n^{-3} b^{-2 s m} \log \left(n^{4} b^{2 s m}\right)\right)=O\left(P n^{-2} b^{-2 s m}\right)=O\left(P n^{-2} b^{-k_{m}+k_{1}}\right) .
\end{aligned}
$$


Let

$B_{P}\left(x, s, \nu, \gamma, \gamma_{1}, \gamma_{2}\right)$

$$
=\#\left\{v \in[0, P) \mid\left\{\left(\left(x+v q_{r}\right)^{2}+\alpha\left(x+v q_{r}\right)+\gamma\right) b^{-k_{m}}\right\} \in\left[\gamma_{1}, \gamma_{2}\right)\right\} .
$$

Corollary 3. Let $0 \leq \gamma_{1}<\gamma_{2} \leq 1$. Under the assumptions of Lemma 8,

$$
B_{P}\left(x, s, t, \nu, \gamma, \gamma_{1}, \gamma_{2}\right)=P\left(\gamma_{2}-\gamma_{1}\right)+O\left(P n^{-2} b^{-k_{m}+k_{1}}\right) .
$$

The proof follows from (10), (86), and Lemma 8 .

Let

$$
\begin{aligned}
B_{P}(x, s, t, j, \nu, g)=\#\left\{v \in[0, P) \mid\left\{\left(\left(x+v q_{r}\right)^{2}+y\right) b^{-k_{m}}\right\}\right. \\
\left.\in\left[\frac{g}{b^{k_{m}-k_{1}+1}}, \frac{g+1}{b^{k_{m}-k_{1}+1}}\right)\right\}
\end{aligned}
$$

where $g \in\left[0, b^{k_{m}-k_{1}+1}\right)$ be integer, $x \in\left[H,(H+1) q_{r}\right)$, and $y=y\left(x+v q_{r}, s\right)$ satisfy $(20)$.

Lemma 9. Under the assumptions of Lemma 8,

$$
B_{P}(x, s, t, j, \nu, g)=P b^{k_{1}-k_{m}-1}\left(1+O\left(n^{-2}\right)\right) \text {. }
$$

Proof. Using Corollary 3 and repeating the proof of Lemma 5, we obtain (89).

Lemma 10. Under the assumptions of Lemma 8, let $x \in E_{\nu s}\left(t, H q_{r}, q_{r}\right) \quad$ if and only if $x+(Q-H) q_{r} \in E_{\nu s}\left(t, Q q_{r}, q_{r}\right)$ for all $Q \in[H, H+P]$. Then

$$
\# A_{j \nu s}\left(t, H q_{r}, P q_{r}\right)=b^{-m} P \# E_{\nu s}\left(t, H q_{r}, q_{r}\right)(1+O(1 / n)) .
$$

Proof. It follows from (52), (37), and the condition of the lemma that

$$
\# A_{j \nu s}\left(t, H q_{r}, P q_{r}\right)=\sum_{x \in E_{\nu s}\left(t, H q_{r}, q_{r}\right)} \# A_{j \nu s x}(P)
$$

with

$$
A_{j \nu s x}(P)=\left\{v \in[0, P) \mid\left(x+v q_{r}\right)^{2}+y=x_{1}+g_{1} b^{k_{1}-1}+x_{2} b^{k_{1}}+\ldots\right.
$$

$$
\left.\ldots+g_{m} b^{k_{m}-1}+x_{m+1} b^{k_{m}}\right\} \text {. }
$$

Hence

$$
\begin{array}{r}
A_{j \nu s x}(P)=\sum_{x_{2}=0}^{b^{k_{2}-k_{1}-1}-1} \ldots \sum_{x_{m}=0}^{b^{k_{m}-k_{m-1}-1}-1}\left\{v \in[0, P) \mid\left(x+v q_{r}\right)^{2}+y\right. \\
\left.=x_{1}+g b^{k_{1}-1}+x_{m+1} b^{k_{m}}\right\}
\end{array}
$$


where

$$
g=g\left(x_{2}, . ., x_{m}\right)=g_{1}+x_{2} b+g_{2} b^{k_{2}-k_{1}}+\ldots+x_{m} b^{k_{m-1}-k_{1}+1}+g_{m} b^{k_{m}-k_{1}} .
$$

Bearing in mind that the condition

$$
x^{2}+y=x_{1}+g b^{k_{1}-1}+x_{m+1} b^{k_{m}}
$$

is equivalent to the condition

$$
\left\{\left(x^{2}+y\right) b^{-k_{m}}\right\} \in\left[\frac{g}{b^{k_{m}-k_{1}+1}}, \frac{g+1}{b^{k_{m}-k_{1}+1}}\right)
$$

with any integer $x_{1} \in\left[0, b^{k_{1}-1}\right), \quad x_{2} \geq 0$ (the integer $g \in\left[0, b^{k_{m}-k_{1}+1}\right.$ ) is fixed), we obtain from (88) that

$$
\begin{aligned}
\# A_{j \nu s x}(P)= & \sum_{x_{2}=0}^{b^{k_{2}-k_{1}-1}-1} \cdots \sum_{x_{m}=0}^{b^{k_{m}-k_{m-1}-1}-1} \#\{v \in[0, P) \mid \\
& \left.\left\{\left(\left(x+v q_{r}\right)^{2}+y\right) b^{-k_{m}}\right\} \in\left[\frac{g}{b^{k_{m}-k_{1}+1}}, \frac{g+1}{b^{k_{m}-k_{1}+1}}\right)\right\} \\
= & \sum_{x_{2}=0}^{b^{k_{2}-k_{1}-1}-1} \cdots \sum_{x_{m}=0}^{b^{k_{m}-k_{m-1}-1}-1} B_{P}(x, s, t, j, \nu, g) .
\end{aligned}
$$

Using Lemma 9 we obtain

$$
\begin{aligned}
\# A_{j \nu s x}(P) & =\sum_{x_{2}=0}^{b^{k_{2}-k_{1}-1}-1} \cdots \sum_{x_{m}=0}^{b^{k_{m}-k_{m-1}-1}-1} P b^{k_{1}-k_{m}-1}\left(1+O\left(n^{-2}\right)\right) \\
& =b^{-m} P\left(1+O\left(n^{-2}\right)\right) .
\end{aligned}
$$

Applying (90) the assertion of Lemma 10 follows.

Let

$$
\beta=b_{r} / q_{r}+\beta_{1} / q_{r}, \text { with }\left|\beta_{1}\right| \leq 1 / 2
$$

where $b_{r}$ is an integer,

$Q^{\prime}=\left\lceil\frac{-q_{r+1} \beta_{1}}{2 n q_{r} \theta_{r}}\right\rceil-2, \quad P_{1}=\left[n^{-7} b^{s^{2} / 2+4 s m}\right], \quad Q_{0}= \begin{cases}Q^{\prime} & \text { if } Q^{\prime} \in\left[0, P_{1}\right) \\ P_{1} & \text { otherwise }\end{cases}$

where $\lceil x\rceil=x+1$ for integer $x$; otherwise $\lceil x\rceil=[x]$.

Lemma 11. Let $q_{r} \leq n^{6} b^{2 s m}, N \in\left[b^{2 n^{2}-5 n}, b^{2 n^{2}}\right), \sqrt{n} \leq t \leq s \leq 2 n-\sqrt{n}$, $x \in\left[0, q_{r}\right), n>m^{2}$. Then $\forall Q \in\left[0, Q_{0}\right)$

$$
x \in E_{\nu s}\left(t, q_{r}\right) \text { if and only if } x+Q q_{r} \in E_{\nu s}\left(t, Q q_{r}, q_{r}\right),
$$

and $\forall Q_{1} \in\left(Q_{0}+2, P_{1}\right]$

(96) $x+P_{1} q_{r} \in E_{\nu s}\left(t, P_{1} q_{r}, q_{r}\right)$ if and only if $x+Q_{1} q_{r} \in E_{\nu s}\left(t, Q_{1} q_{r}, q_{r}\right)$. 
Proof. Consider the case of $\theta_{r}>0$. (For $\theta_{r}<0$ the proof is similar.) Let

$$
z(x) \equiv p_{r}(2 n x+s)+b_{r}\left(\bmod 2 n q_{r}\right), \quad \text { with } z(x) \in\left[0,2 n q_{r}\right)
$$

and

$$
\gamma=\gamma(x)=\beta_{1}+\frac{\theta_{r}(2 n x+s)}{q_{r+1}} .
$$

Using (57), (93) and the condition of lemma, we have that for sufficiently large $n$.

$$
|\gamma(x)|<3 / 4
$$

It follows from $(31),(56),(93),(97)$, and (98) that

$$
\begin{aligned}
\alpha_{x+Q q_{r}, s} & =\left\{\left(\frac{p_{r}}{q_{r}}\left(2 n\left(x+Q q_{r}\right)+s\right)+\frac{\theta_{r}}{q_{r} q_{r+1}}\left(2 n\left(x+Q q_{r}\right)+s\right)\right) / 2 n+\frac{\beta}{2 n}\right\} \\
& =\left\{\frac{p_{r}(2 n x+s)+b_{r}+\beta_{1}}{2 n q_{r}}+\frac{\theta_{r}\left(2 n\left(x+Q q_{r}\right)+s\right)}{2 n q_{r} q_{r+1}}\right\} \\
(100) & =\left\{\frac{z(x)+\gamma(x)}{2 n q_{r}}+\frac{\theta_{r} Q}{q_{r+1}}\right\} .
\end{aligned}
$$

Applying (37) we have

$$
x+Q q_{r} \in E_{\nu s}\left(t, Q q_{r}, q_{r}\right) \text { if and only if } \alpha_{x+Q q_{r}, s} \in \Delta_{t, \nu}
$$

or

$$
\left\{\frac{z(x)+\gamma(x)}{2 n q_{r}}+\frac{\theta_{r} Q}{q_{r+1}}\right\} \in\left[\frac{t+\xi_{\nu}}{2 n}, \frac{t+\xi_{\nu+1}}{2 n}\right) .
$$

Now let

(102) $x+Q q_{r} \in E_{\nu s}\left(t, Q q_{r}, q_{r}\right)$ and $x+(Q+1) q_{r} \notin E_{\nu}\left(t,(Q+1) q_{r}, q_{r}\right)$

with any $Q \in\left[0, Q_{0}\right]$. Using (101) and that $\theta_{r}>0$ and $t \leq 2 n-\sqrt{n}$, we get that the transition $Q \rightarrow Q+1$ causes a shift in the fractional part of (101) to the right of the interval $\left[\frac{t+\xi_{\nu}}{2 n}, \frac{t+\xi_{\nu+1}}{2 n}\right)$ to the interval $\left[\frac{t+\xi_{\nu+1}}{2 n}, \frac{t+\xi_{\nu+2}}{2 n}\right)$ :

$$
\left\{\frac{z(x)+\gamma(x)}{2 n q_{r}}+\frac{\theta_{r} Q}{q_{r+1}}\right\}<\frac{t+\xi_{\nu+1}}{2 n} \leq\left\{\frac{z(x)+\gamma(x)}{2 n q_{r}}+\frac{\theta_{r}(Q+1)}{q_{r+1}}\right\} .
$$

We have from (57), (94), (99) and the condition of lemma that

$$
0 \leq Q \leq P_{1} \leq n^{-7} b^{s^{2} / 2+4 s m}<\frac{q_{r+1}}{8 n q_{r}}
$$

and

$$
\left|\frac{\gamma(x)}{2 n q_{r}}+\frac{\theta_{r} Q}{q_{r+1}}\right|<\frac{1}{2 n q_{r}} .
$$


Bearing in mind (97), (101) and so that $t \in[\sqrt{n}, 2 n-\sqrt{n}]$, we get

$$
\left\{\frac{z(x)+\gamma(x)}{2 n q_{r}}+\frac{\theta_{r} Q}{q_{r+1}}\right\} \in\left[\frac{\sqrt{n}}{2 n}, \frac{2 n-\sqrt{n}+2}{2 n}\right],
$$

and we can eliminate the sign of the fractional part in (103):

Hence

$$
\frac{z(x)+\gamma(x)}{2 n q_{r}}+\frac{\theta_{r} Q}{q_{r+1}}<\frac{t+\xi_{\nu+1}}{2 n} \leq \frac{z(x)+\gamma(x)}{2 n q_{r}}+\frac{\theta_{r}(Q+1)}{q_{r+1}} .
$$

$$
Q=\left\lceil\frac{q_{r+1}}{\theta_{r}}\left(\frac{t+\xi_{\nu+1}}{2 n}-\frac{z(x)+\gamma(x)}{2 n q_{r}}\right)\right\rceil-1 .
$$

Let $\xi_{\nu+1}=1-\{\alpha i\}=1-\left\{\frac{p_{r} i}{q_{r}}+\frac{\theta_{r} i}{q_{r} q_{r+1}}\right\}=\frac{i_{1}}{q_{r}}-\frac{\theta_{r} i}{q_{r} q_{r+1}}$ with the same $i \in[0, m], i_{1} \in\left[1, q_{r}\right]$. It follows from (106), (97) and (98) that

$$
\begin{aligned}
Q & =\left\lceil\frac{q_{r+1}}{\theta_{r}}\left(\frac{q_{r} t+i_{1}}{2 n q_{r}}-\frac{z(x)+\beta_{1}}{2 n q_{r}}-\frac{\theta_{r}(2 n x+s+i)}{2 n q_{r} q_{r+1}}\right)\right\rceil-1 \\
& =\left\lceil\frac{q_{r+1}}{2 n q_{r} \theta_{r}}\left(v-\beta_{1}\right)-\frac{(2 n x+s+i)}{2 n q_{r}}\right\rceil-1,
\end{aligned}
$$

with $v=q_{r} t-z(x)+i_{1}$. From the condition of the lemma we have that $0 \leq 2 n x+s+i<2 n q_{r}-\sqrt{n}+m<2 n q_{r}$. Using (93) we get, that if $v \neq 0$, then

$$
|Q| \geq \frac{q_{r+1}}{4 n q_{r} \theta_{r}}-3 \geq \frac{q_{r+1}}{4 n q_{r}}-3 .
$$

This is in contradiction with (104).

Let $v=0$. We get from (94) and (107) that

$$
Q=Q(x)=\left\lceil\frac{-q_{r+1} \beta_{1}}{2 n q_{r} \theta_{r}}-\frac{2 n x+s+i}{2 n q_{r}}\right\rceil-1 \in\left[Q^{\prime}, Q^{\prime}+2\right],
$$

where $i \in[0, m]$. Hence if $Q^{\prime}<-2$ or $Q^{\prime}>P_{1}$, then there is no $Q \in\left[0, P_{1}\right]$ satisfying (102). Now, let (109) $x+Q_{1} q_{r} \in E_{\nu}\left(t, Q_{1} q_{r}, q_{r}\right)$ and $x\left(Q_{1}-1\right) q_{r} \notin E_{\nu}\left(t,\left(Q_{1}-1\right) q_{r}, q_{r}\right)$, with $0 \leq Q_{1}=Q_{1}(x) \leq P_{1}$. Similarly to (94)-(108) we obtain for all $x \in\left[0, q_{r}\right)$, that

$$
Q_{1}(x) \in\left[Q^{\prime}, Q^{\prime}+2\right] .
$$

Hence if $Q^{\prime}<-2$ or $Q^{\prime}>P_{1}$, then there is no $Q_{1}$ satisfying (109). Now from (94), (102), (108), (109), and (110), the assertion of Lemma 11 follows.

Lemma 12. Under the assumptions of Lemma 11,

$$
\# A_{j \nu s}(t, N)=b^{-m} \# E_{\nu s}(t, N)(1+O(1 / n))+O\left(N / n^{4}\right) .
$$

Proof. Let

$$
P_{2}=\left[n^{4} b^{s^{2} / 2+3 s m}\right]+1 .
$$


We have from (94) that

$$
P_{2}=O\left(n^{-2} P_{1}\right)
$$

If $Q_{0} \leq P_{2}$, or $Q_{0} \in\left[P_{1}-P_{2}, P_{1}\right]$, then we use the trivial estimate

$$
\# A_{j \nu s}\left(t, Q_{0} q_{r}\right) \leq \# E_{\nu s}\left(t, Q_{0} q_{r}\right) \leq P_{2} q_{r}
$$

for $Q_{0} \leq P_{2}$, and

$$
\# A_{j \nu s}\left(t, Q_{0} q_{r},\left(P_{1}-Q_{0}\right) q_{r}\right) \leq \# E_{\nu s}\left(t, Q_{0} q_{r},\left(P_{1}-Q_{0}\right) q_{r}\right) \leq P_{2} q_{r}
$$
for $Q_{0} \in\left[P_{1}-P_{2}, P_{1}\right]$. Let $Q_{0} \leq P_{2}$.

Applying (94), (111), (112), Lemma 11, and Lemma 10 with $H=P_{2}$ and $P=P_{1}-P_{2}$, we obtain that

$$
\# E_{\nu, s}\left(t, P_{2} q_{r},\left(P_{1}-P_{2}\right) q_{r}\right)=\left(P_{1}-P_{2}\right) \# E_{\nu, s}\left(t, P_{2} q_{r}, q_{r}\right)
$$

and

$\# A_{j \nu s}\left(t, P_{2} q_{r},\left(P_{1}-P_{2}\right) q_{r}\right)=b^{-m}\left(P_{1}-P_{2}\right) \# E_{\nu s}\left(t, P_{2} q_{r}, q_{r}\right)(1+O(1 / n))$.

Hence

$\# A_{j \nu s}\left(t, P_{2} q_{r},\left(P_{1}-P_{2}\right) q_{r}\right)=b^{-m} \# E_{\nu s}\left(t, P_{2} q_{r},\left(P_{1}-P_{2}\right) q_{r}\right)(1+O(1 / n))$.

Using (45) and (113) we have

$$
\# A_{j \nu s}\left(t, P_{1} q_{r}\right)=b^{-m} \# E_{\nu s}\left(t, P_{1} q_{r}\right)(1+O(1 / n))+O\left(P_{2} q_{r}\right) .
$$

By (114) and Lemma 10 with $H=0$ and $P=P_{1}-P_{2}$, we get similarly that $(115)$ is valid for the case $Q_{0} \in\left[P_{1}-P_{2}, P_{1}\right]$. Now we prove that (115) is valid for $Q_{0} \in\left(P_{2}, P_{1}-P_{2}\right)$. Let $Q_{0} \in\left(P_{2}, P_{1}-P_{2}\right)$. We have from Lemma 11 that

$$
\# E_{\nu s}\left(t, Q_{0} q_{r}\right)=Q_{0} \# E_{\nu s}\left(t, q_{r}\right)
$$

and

$$
\begin{aligned}
\# E_{\nu s}\left(t,\left(Q_{0}+3\right) q_{r},\left(P_{1}-Q_{0}-3\right) q_{r}\right)= & \left(P_{1}-Q_{0}-3\right) \# E_{\nu s}\left(t, P_{1} q_{r}, q_{r}\right) \\
= & \left(P_{1}-Q_{0}-3\right) \\
& \times \# E_{\nu s}\left(t,\left(Q_{0}+3\right) q_{r}, q_{r}\right) .
\end{aligned}
$$

Hence

$$
\begin{aligned}
\# E_{\nu s}\left(t, P_{1} q_{r}\right)= & Q_{0} \# E_{\nu s}\left(t, q_{r}\right) \\
& +\left(P_{1}-Q_{0}-3\right) \# E_{\nu s}\left(t,\left(Q_{0}+3\right) q_{r}, q_{r}\right)+3 \epsilon q_{r} .
\end{aligned}
$$

Applying (94), (96) and Lemma 10 twice, we have that

$$
\# A_{j \nu s}\left(t, Q_{0} q_{r}\right)=b^{-m} Q_{0} \# E_{\nu s}\left(t, q_{r}\right)(1+O(1 / n)),
$$


and

$$
\begin{aligned}
& \# A_{j \nu s}\left(t,\left(Q_{0}+3\right) q_{r},\left(P_{1}-Q_{0}-3\right) q_{r}\right) \\
& =b^{-m}\left(P_{1}-Q_{0}-3\right) \# E_{\nu s}\left(t,\left(Q_{0}+3\right) q_{r}, q_{r}\right)(1+O(1 / n)) .
\end{aligned}
$$

Hence, and from (116), we get

$$
\begin{array}{r}
\# A_{j \nu s}\left(t, P_{1} q_{r}\right)=b^{-m}\left(Q_{0} \# E_{\nu s}\left(t, q_{r}\right)+\left(P_{1}-Q_{0}-3\right) \# E_{\nu s}\left(t, P_{1} q_{r}, q_{r}\right)\right) \\
\times(1+O(1 / n))+3 \epsilon q_{r}=b^{-m} \# E_{\nu s}\left(t, P_{1} q_{r}\right)(1+O(1 / n))+O\left(q_{r}\right) .
\end{array}
$$

Therefore (115) is valid for all $Q_{0} \in\left[0, P_{1}\right]$. Bearing in mind that (115) is valid for all $\beta$, we get (replacing $\beta$ by $\beta+i P_{1} q_{r} \alpha$ )

$\# A_{j \nu s}\left(t, i P_{1} q_{r}, P_{1} q_{r}\right)=b^{-m} \# E_{\nu s}\left(t, i P_{1} q_{r}, P_{1} q_{r}\right)(1+O(1 / n))+O\left(P_{2} q_{r}\right)$, with $i=0,1, \ldots$. Hence

$$
\begin{aligned}
\# A_{j \nu s}(t, N) & =\sum_{0 \leq i<\left[N / P_{1} q_{r}\right]} \# A_{j \nu s}\left(t, i P_{1} q_{r}, P_{1} q_{r}\right)+O\left(P_{1} q_{r}\right) \\
& =\sum_{0 \leq i<\left[N / P_{1} q_{r}\right]}\left(b^{-m} \# E_{\nu s}\left(t, i P_{1} q_{r}, P_{1} q_{r}\right)\right. \\
& \left.\times(1+O(1 / n))+O\left(P_{2} q_{r}\right)\right)+O\left(P_{1} q_{r}\right) .
\end{aligned}
$$

By (94), (112) and condition of the lemma, we obtain

$$
\begin{aligned}
A_{j \nu s}(t, N) & =b^{-m} E_{\nu s}(t, N)(1+O(1 / n))+O\left(P_{1} q_{r}+N / n^{4}\right) \\
& =b^{-m} E_{\nu s}(t, N)(1+O(1 / n))+O\left(N / n^{4}\right) .
\end{aligned}
$$

Lemma 13. Let $s_{0}, s_{0}+s_{1} \in[0,2 n)$. Then

$$
\sum_{s_{0} \leq s<s_{0}+s_{1}} \sum_{0 \leq t<2 n} \sum_{0 \leq \nu \leq m} \# E_{\nu s}(t, N) \leq s_{1} N .
$$

Proof. From (15), (31), and (35) we have that

$$
\sum_{0 \leq t<2 n} \sum_{0 \leq \nu \leq m} \# E_{\nu s}(t, N)=N .
$$

Lemma 14. Let $\left[t_{0}, t_{0}+t_{1}\right) \in[0,2 n)$. Then

$$
\mu=\sum_{0 \leq s<2 n} \sum_{t_{0} \leq t<t_{0}+t_{1}} \sum_{0 \leq \nu \leq m} \# E_{\nu s}(t, N)=O\left(t_{1} N\right) .
$$

Proof. From (15), (31), and (35) we have that

$$
\begin{aligned}
\sum_{t_{0} \leq t<t_{0}+t_{1}} \sum_{0 \leq \nu \leq m} \# E_{\nu s}(t, N) & \\
& =\#\left\{x \in[0, N) \mid\left\{\frac{\alpha(2 n x+s)+\beta}{2 n}\right\} \in\left[\frac{t_{0}}{2 n} \frac{t_{0}+t_{1}}{2 n}\right)\right\},
\end{aligned}
$$


and

$$
\mu=\sum_{x=0}^{N-1} \#\left\{s \in[0,2 n) \mid\left\{\frac{\alpha(2 n x+s)+\beta}{2 n}\right\} \in\left[\frac{t_{0}}{2 n}, \frac{t_{0}+t_{1}}{2 n}\right)\right\} .
$$

Since $\alpha \in(0,1)$, we have that

$$
\#\left\{s \in[0,2 n) \mid\left\{\frac{\alpha(2 n x+s)+\beta}{2 n}\right\} \in\left[\frac{t_{0}}{2 n}, \frac{t_{0}+t_{1}}{2 n}\right)\right\} \leq \frac{t_{1}}{\alpha}+2 .
$$

Hence from (117) we get the assertion of the lemma.

Lemma 15. Let $N \in\left[b^{2 n^{2}-5 n}, b^{2 n^{2}}\right)$. Then

$$
\# V_{n}^{\prime}\left(N, G_{m}\right)=b^{-m} \# U_{n}^{\prime}(N)(1+O(1 / \sqrt{n}))+O(N \sqrt{n}) .
$$

Proof. Using (44) we have that

$$
\# V_{n}^{\prime}\left(N, G_{m}\right)=\mu_{1}+\epsilon_{2} \mu_{2}+\epsilon_{3} \mu_{3},
$$

with $0 \leq \epsilon_{2}, \epsilon_{3} \leq 1$, and

$$
\begin{aligned}
\mu_{1} & =\sum_{\sqrt{n} \leq t \leq s<2 n-\sqrt{n}} \sum_{0 \leq \nu \leq m} \sum_{0 \leq j \leq l_{1}(\nu)} \# A_{j \nu s}(t, N) \\
\mu_{2} & =\sum_{0 \leq s<\sqrt{n}} \sum_{0 \leq t<2 n-\sqrt{n}} \sum_{0 \leq \nu \leq m} \sum_{0 \leq j \leq l_{1}(\nu)} \# A_{j \nu s}(t, N) \\
\mu_{3} & =\sum_{0 \leq s<2 n-\sqrt{n}} \sum_{0 \leq t<\sqrt{n}} \sum_{0 \leq \nu \leq m} \sum_{0 \leq j \leq l_{1}(\nu)} \# A_{j \nu s}(t, N)
\end{aligned}
$$

Now let

$$
\begin{aligned}
\sigma_{2} & =\sum_{0 \leq s<\sqrt{n}} \sum_{0 \leq t<2 n-\sqrt{n}} \sum_{0 \leq \nu \leq m} \sum_{0 \leq j \leq l_{1}(\nu)} \# E_{j \nu s}(t, N), \\
\sigma_{3} & =\sum_{0 \leq s<2 n-\sqrt{n}} \sum_{0 \leq t<\sqrt{n}} \sum_{0 \leq \nu \leq m} \sum_{0 \leq j \leq l_{1}(\nu)} \# E_{\nu s}(t, N) .
\end{aligned}
$$

By Lemma 6 and Lemma 12 we get

$$
\begin{aligned}
\mu_{1}= & \sum_{\sqrt{n} \leq t \leq s<2 n-\sqrt{n}} \sum_{0 \leq \nu \leq m} \sum_{0 \leq j \leq l_{1}(\nu)}\left(b^{-m} \# E_{\nu s}(t, N)\right. \\
& \left.\times(1+O(1 / n))+O\left(N n^{-4}\right)\right) .
\end{aligned}
$$

Using (16) and Lemma 13, we get

$$
\mu_{2} \leq \sigma_{2} \leq 4 \sqrt{n}(\alpha+1) N .
$$

Applying Lemma 14, we obtain

$$
\mu_{3} \leq \sigma_{3} \leq(\alpha+1)(\sqrt{n}+3) \alpha^{-1} N .
$$


From (118)-(121), we have

$$
\begin{array}{r}
\# V_{n}^{\prime}\left(N, G_{m}\right)=\sum_{0 \leq t \leq s<2 n-\sqrt{n}} \sum_{0 \leq \nu \leq m} \sum_{0 \leq j \leq l_{1}(\nu)}\left(b^{-m} \# E_{\nu s}(t, N)(1+O(1 / n))\right. \\
\left.+O\left(N n^{-4}\right)\right)+\epsilon_{2} \mu_{2}+\epsilon_{3} \mu_{3}+\epsilon_{4} \sigma_{2}+\epsilon_{5} \sigma_{3},
\end{array}
$$

with $0 \leq\left|\epsilon_{\nu}\right| \leq 1, \nu \in[2,5]$. By (36), (122), and (123) we obtain the assertion of Lemma 15.

Main Lemma. Let $N \in\left[b^{2 n^{2}-5 n}, b^{2 n^{2}}\right)$. Then

$$
\# V_{n}\left(N, G_{m}\right)=b^{-m} \# U_{n}(N)(1+O(1 / \sqrt{n})) \text {. }
$$

Proof. Using (5), (23), (34), (39), (40), (42) and repeating the proof of Lemmas $2-15$ for the case $t>s$, we obtain

$$
\begin{aligned}
\# U_{n}(N) & =\# U_{n}^{*}(N)+O(N \sqrt{n})=\# U_{n}^{\prime}(N)+O(N \sqrt{n}), \\
\# V_{n}\left(N, G_{m}\right) & =\# V_{n}^{*}\left(N, G_{m}\right)+O(N \sqrt{n})=\# V_{n}^{\prime}\left(N, G_{m}\right)+O(N \sqrt{n}),
\end{aligned}
$$

and

$$
\# V_{n}\left(N, G_{m}\right)=b^{-m} \# U_{n}(N)(1+O(1 / \sqrt{n}))+O(N \sqrt{n}) .
$$

Applying (22) we get (124).

Remark. For the case $\alpha>1$, we consider the line $(z, \alpha z+\beta)$ as the line $((w-\beta) / \alpha, w)$; the variable $y$ as an independent variable (instead of $x)$; and in $(5),(6)$ we choose the case of $y>x$. (Hence we use the formula $u=y^{2}+2 y-x$ instead of $\left.u=x^{2}+y\right)$. Now repeating the proof of Lemmas 2-15, we get the proof of the Main Lemma for the case $\alpha>1$.

\subsection{The case of the rational line with $\alpha>0$.}

In this subsection we slightly modify partition of Section 3.1. Next we prove that the estimate of Lemma 10 can be applied for rational $\alpha$.

Let $\alpha=p / q>0,(p, q)=1$. We use the notation of (15), (16):

$$
\xi_{j}=j / q, j=0, \ldots, q ; \quad f(i)=q(1-\{\alpha i\}), i=1, \ldots, m .
$$

We fix an integer $\nu \in[0, q-1]$ and consider the sequence $\left(l_{i}(\nu)\right)_{i=1}^{m}$ :

$$
l_{i}(\nu)= \begin{cases}{[\alpha i],} & \text { if } \nu<f(i) \\ {[\alpha i]+1 .} & \text { otherwise. }\end{cases}
$$

Lemma 16. Let

$$
\left\{\frac{\alpha(2 n x+s)+\beta}{2 n}\right\} \in\left[\frac{t+\nu / q}{2 n}, \frac{t+(\nu+1) / q}{2 n}\right), \text { with } \nu \in[0, q-1]
$$

Then

$$
\left.\left\{\frac{\alpha(2 n x+s+i)+\beta}{2 n}\right\} \in\left[\frac{t+l_{i}(\nu)}{2 n}, \frac{t+l_{i}(\nu)+1}{2 n}\right), \quad i=1, \ldots, m .\right)
$$


Proof. Using (126) we get

$$
\begin{aligned}
z_{i} & =\left\{\frac{\alpha(2 n x+s+i)+\beta}{2 n}\right\}=\left\{\frac{\alpha(2 n x+s)+\beta}{2 n}+\frac{\alpha i}{2 n}\right\} \\
& =\left\{\frac{t+\nu / q+\epsilon+[\alpha i]+\{\alpha i\}}{2 n}\right\}, \quad \text { with } 0 \leq \epsilon<1 / q .
\end{aligned}
$$

From here and from (125), we have that

$$
z_{i}=\left\{\frac{t+[\alpha i]+\nu / q+1-f(i) / q+\epsilon}{2 n}\right\} .
$$

It is evident that if $f(i)>\nu$, then

$$
\nu / q+\epsilon-f(i) / q<(\nu+1) / q-f(i) / q \leq 0 .
$$

Applying (126) we get (127) with $l_{i}(\nu)=[\alpha i]$.

Now let $f(i) \leq \nu$. Then

$$
0 \leq \nu / q+\epsilon-f(i) / q<(\nu+1) / q
$$

By (126) we obtain (127) with $l_{i}(\nu)=[\alpha i]+1$.

We use a variant of notations (31) - (43) as follows :

$$
\begin{aligned}
\alpha_{x s} & =\{p(2 n x+s) / q+\beta\} \\
\Delta_{t, \nu} & =\left[\frac{t+\xi_{\nu}}{2 n}, \frac{t+\xi_{\nu+1}}{2 n}\right), \\
w_{x s} & = \begin{cases}1 & \text { if } \alpha_{x s}=0 \\
0 & \text { otherwise }\end{cases}
\end{aligned}
$$

and in the summation of $y$ in formulas (34), (36), (42), and (44), we apply $l_{1}^{\prime}(\nu)=l_{1}(\nu)-w_{x s}$ instead of $l_{1}(\nu)$. (Note that $w_{x s}=0$ for all $\beta \neq c / q$ with integer $c$.) For fixed integers $x, s, t, \nu$ and $j$, we denote: $\left(k_{n}\right)_{n \geq 1}$ as an increasing sequence of integers from the set

$$
\begin{aligned}
\left\{\left(s+i^{\prime}\right)^{2}+t+l_{i^{\prime}}(\nu)+v+1 \mid v\right. & =j \delta_{i^{\prime}}, j \delta_{i^{\prime}}+1, \ldots, l_{i^{\prime}+1}(\nu)-l_{i^{\prime}}(\nu)-w_{x s} \\
i^{\prime} & =0,1, \ldots, m-1\}
\end{aligned}
$$

and $\left(z^{(r)}(x, s, j)\right)_{r=1}^{m}$ as the sequence of the first $m$ vectors from the set

$$
\left(\left(2 n x+s+i^{\prime},[\alpha(2 n x+s)+\beta]+l_{i^{\prime}}(\nu)+v\right)\right)
$$


with $i^{\prime}=0,1, \ldots, m$, and

$$
\begin{aligned}
& v=j \delta_{i^{\prime}}, j \delta_{i^{\prime}}+1, \ldots, l_{i^{\prime}+1}(\nu)-l_{i^{\prime}}(\nu)-w_{x s}, \quad(\text { see }(47)) ; \\
& E_{\nu s}\left(x^{\prime}, t, N\right)=\left\{x \in[0, N) \mid x \equiv x^{\prime}(\bmod q), \alpha_{x, s} \in \Delta_{t, \nu}\right\}, \\
& A_{j \nu s}\left(x^{\prime}, t, N\right)=\left\{x \in[0, N) \mid x \equiv x^{\prime}(\bmod q),\right. \\
& \left.\chi_{\omega_{n} G_{m}}(z(x, s, j))=1, \quad \alpha_{x, s} \in \Delta_{t, \nu}\right\}
\end{aligned}
$$

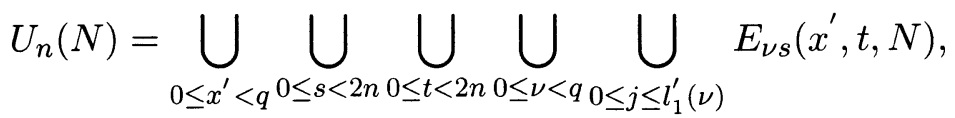

where $l_{1}^{\prime}(\nu)=l_{1}(\nu)-w_{x^{\prime} s}$,

$$
V_{n}\left(N, G_{m}\right)=\bigcup_{0 \leq x^{\prime}<q} \bigcup_{0 \leq s<2 n} \bigcup_{0 \leq t<2 n} \bigcup_{0 \leq \nu<q} \bigcup_{0 \leq j \leq l_{1}^{\prime}(\nu)} A_{j \nu s}\left(x^{\prime}, t, N\right) .
$$

Lemma 17. Let $N \in\left[b^{2 n^{2}-5 n}, b^{2 n^{2}}\right), \quad \sqrt{n} \leq t \leq s \leq 2 n-\sqrt{n}$. Then

$$
\# A_{j \nu s}\left(x^{\prime}, t, N\right)=b^{-m} \# E_{\nu s}\left(x^{\prime}, t, N\right)(1+O(1 / n)) \text {. }
$$

Proof. Let

$$
\delta(x, s, t, \nu)= \begin{cases}1 & \text { if } \alpha_{x, s} \in \Delta_{t, \nu} \\ 0 & \text { otherwise }\end{cases}
$$

It is easy to see that the sequences $\alpha_{x, s}, \delta(x, s, t, \nu) \quad(x=1,2, \ldots)(128)$ (129) have period $q$. Hence we have from (132)

$$
\# E_{\nu s}\left(x^{\prime}, t, N\right)=\delta\left(x^{\prime}, s, t, \nu\right)(N / q+\epsilon), \quad \text { with }|\epsilon| \leq 1 .
$$

Consider Lemma 10 with $q_{r}=q(r=1)$. Then the condition of Lemma 10

$$
x \in E_{\nu s}\left(t, H q_{r}, q_{r}\right) \text { if and only if } x+Q q_{r} \in E_{\nu s}\left(t, Q q_{r}, q_{r}\right)
$$

for all $Q \in[H, H+P)$ is valid for all $H \geq 0$ and all $P \geq 1$. Applying (133), and $(91),(92)$ for the case $P=[N / q], x \equiv x^{\prime}(\bmod q)$, we obtain

$$
\# A_{j \nu s}\left(x^{\prime}, t, N\right)=b^{-m} \delta\left(x^{\prime}, s, t, \nu\right)[N / q](1+O(1 / n)) \text {. }
$$

(136) follows from (137) and the condition of the lemma

(Def. $V_{n}^{\prime}\left(U_{n}^{\prime}\right)$ be the part of $V_{n}\left(U_{n}\right)$ with $t<s$ )

Lemma 18. Let $N \in\left[b^{2 n^{2}-5 n}, b^{2 n^{2}}\right)$. Then

$$
\# V_{n}^{\prime}\left(N, G_{m}\right)=b^{-m} \# U_{n}^{\prime}(N)(1+O(1 / n))+O(N \sqrt{n}) .
$$

Proof. Using Lemmas 13, 14, 17 and repeating the proof of Lemma 15, we obtain (138).

Now we consider the case of $t \geq s$ and (similarly to (138)), we get: 
Corollary. Let $N \in\left[b^{2 n^{2}-5 n}, b^{2 n^{2}}\right)$. Then

$$
\# V_{n}\left(N, G_{m}\right)=b^{-m} \# U_{n}(N)(1+O(1 / \sqrt{n})) \text {. }
$$

The proof is the same as that of the Main Lemma.

\subsection{The case of the horizontal lines $(\alpha=0)$.}

Using (19)-(24) we obtain that $\beta \geq 0, y=0, t=[\beta], U_{n}(N)=2 n N$. Let $N \in\left[b^{2 n^{2}-5 n}, b^{2 n^{2}}\right)$. It follows from (5), (6) that for $s \in[t, 2 n-4 m)$ the block of digits $G_{m}=\left(g_{1}, \ldots, g_{m}\right)$ appears at the point $(2 n x+s, t)$ if and only if

$$
\begin{aligned}
x^{2}=x_{1}+g_{1} b^{s^{2}+t}+x_{2} b^{s^{2}+t+1} & +g_{2} b^{(s+1)^{2}+t} \\
& +\ldots+g_{m} b^{(s+m-1)^{2}+t}+x_{m+1} b^{(s+m-1)^{2}+t+1},
\end{aligned}
$$

where $x_{i} \in\left[0, b^{k_{i}-k_{i-1}-1}\right)$ are integers $(i=1, \ldots, m), k_{0}=0$, and $k_{i}=$ $(s+i-1)^{2}+t+1, i=1,2, \ldots$. We have from (39) that

$$
\begin{aligned}
& \# V_{n}\left(N, G_{m}\right)=\sum_{s \in[t, 2 n-4 m)} \sum_{x_{2}=0}^{b^{k_{2}-k_{1}-1}-1} \cdots \sum_{x_{m}=0}^{b^{k_{m}-k_{m-1}-1}-1} \#\{x \in[0, N) \mid \\
& \left.\left\{x^{2} b^{-k_{m}}\right\} \in\left[\frac{g}{b^{k_{m}-k_{1}+1}}, \frac{g+1}{b^{k_{m}-k_{1}+1}}\right)\right\}+O(N),
\end{aligned}
$$

with

$$
g=g\left(x_{2}, . ., x_{m}\right)=g_{1}+x_{2} b+g_{2} b^{k_{2}-k_{1}}+\ldots+x_{m} b^{k_{m-1}-k_{1}+1}+g_{m} b^{k_{m}-k_{1}} .
$$

Using (88) and Lemma 9 with $q_{r}=1, x=0, H=0$ and $P=N$, we have that

$$
\# V_{n}\left(N, G_{m}\right)=\sum_{s \in[t, 2 n-4 m)} \sum_{x_{2}=0}^{b^{k_{2}-k_{1}-1}-1} \cdots \sum_{x_{m}=0}^{b^{k_{m}-k_{m-1}-1}-1} N b^{k_{1}-k_{m}-1}\left(1+O\left(n^{-1}\right)\right) \text {. }
$$

Bearing in mind that $U_{n}(N)=2 n N$, we have that

$$
\# V_{n}\left(N, G_{m}\right)=b^{-m} \# U_{n}(N)(1+O(1 / \sqrt{n})) \text {. }
$$

\subsection{The case of vertical lines.}

Using (19)-(24), we get $x=0, s=[\beta]$, and $U_{n}(N)=2 n N$. Let $t \in$ $[s, 2 n-m), N \in\left[b^{2 n^{2}-5 n}, b^{2 n^{2}}\right)$. The block of digits $G_{m}=\left(g_{1}, \ldots, g_{m}\right)$ appears at the point $(s, 2 n y+t)$ if and only if

$$
\begin{aligned}
y^{2}+2 y & =y_{1}+g_{1} b^{t^{2}+2 t-s}+y_{2} b^{t^{2}+2 t-s+1}+g_{2} b^{(t+1)^{2}+2 t-s+2} \\
& +\ldots+g_{m} b^{(t+m-1)^{2}+2(t+m-1)-s}+y_{m+1} b^{(t+m-1)^{2}+2(t+m-1)-s+1},
\end{aligned}
$$


where $y_{i+1} \in\left[0, b^{2 t+2 i}\right)$ are integers $i=1,2, \ldots$ Repeating the previous proof, we get

$$
\# V_{n}\left(N, G_{m}\right)=b^{-m} \# U_{n}(N)(1+O(1 / \sqrt{n})) .
$$

\subsection{Completion of the proof of Theorem 1 .}

In this subsection we go from the configuration $\omega_{n}$ to the configuration $\omega_{\infty}$.

Let $P \geq 1$ be an integer. There exists an integer $n \geq 1$ such that

$$
2(n-1) b^{2(n-1)^{2}} \leq P<2 n b^{2 n^{2}} .
$$

Let $N=[P / 2 n], P=2 n N+N_{1}$, where $N_{1} \in[0,2 n), \alpha>0$ is irrational. Let $U(P)$ be the subset of all lattice points $(u, v)$ such that the line $(z, \alpha z+$ $\beta)_{z \in[0, P)}$ intersects the square $(u, v)+[0,1)^{2}$. Applying the definition of $U_{n}(N)$ (see (21)-(24)), we have that

$$
\# U(P)=\# U_{n}(N)+O(n) .
$$

Let

$$
V\left(P, G_{m}\right)=\left\{z \in U(P) \mid G_{m}=\left(\omega\left(z^{(1)}\right), \ldots, \omega\left(z^{(m)}\right)\right)\right\}
$$

be the set of the linear indices of the beginning of a block $G_{m}$ in the linear configuration of digits, confined to the $P$-square configuration. It is evident, that

$$
\# V\left(P, G_{m}\right)=\# V\left(2 n N, G_{m}\right)+O(n),
$$

with

$$
V\left(2 n N, G_{m}\right)=\left\{z \in U_{n}(N) \mid G_{m}=\left(\omega\left(z^{(1)}\right), \ldots, \omega\left(z^{(m)}\right)\right)\right\} .
$$

To compute $\# V\left(2 n N, G_{m}\right)$, consider the configuration $\omega$ as the union of the parts of the configurations $\omega_{n}, \omega_{n-1}$ and $\omega_{n-2}^{\prime}$ (where $\omega_{n-2}^{\prime}$ is the part of configuration $\omega$ corresponding to $\omega_{n-2}$ ). We use a trivial estimate for $\omega_{n-2}^{\prime}$ :

By (8) (definition of $\omega),(34),(39)$, and (145), we have

$$
\begin{aligned}
\# V\left(2 n N, G_{m}\right)= & \# V_{n}\left(N, G_{m}\right)-\# V_{n}\left(\left[(n-1) b^{2(n-1)^{2}} / n\right], G_{m}\right) \\
& +\# V_{n-1}\left(b^{2(n-1)^{2}}, G_{m}\right)+O\left(n b^{2(n-2)^{2}}\right),
\end{aligned}
$$

and

$$
\# U_{n-1}\left(b^{2(n-1)^{2}}\right)=\# U_{n}\left(\left[(n-1) b^{2(n-1)^{2}} / n\right]\right)+O(n) .
$$

Let $\alpha$ be irrational. Using the Main Lemma, we get

$$
\begin{aligned}
\# V\left(2 n N, G_{m}\right)= & b^{-m} \# U_{n}(N)(1+O(1 / \sqrt{n})) \\
& -b^{-m} \# U_{n}\left(\left[(n-1) b^{2(n-1)^{2}} / n\right]\right)(1+O(1 / \sqrt{n})) \\
& +b^{-m} \# U_{n-1}\left(b^{2(n-1)^{2}}\right)(1+O(1 / \sqrt{n}))+O\left(n b^{2(n-2)^{2}}\right) .
\end{aligned}
$$


From here, (22), and (146), we obtain

$$
\# V\left(2 n N, G_{m}\right)=b^{-m} \# U_{n}(N)(1+O(1 / \sqrt{n})) \text {. }
$$

Hence from (144), (143), and (142), we obtain

$$
\# V\left(P, G_{m}\right)=b^{-m} \# U(P)\left(1+O\left(1 /(\log P)^{1 / 4}\right)\right. \text {. }
$$

For the case $\alpha>0$ rational (the horizontal line, and vertical lines) we use (139) (respectively (140), (141)) instead of the Main Lemma. Now by (3) we obtain the assertion of Theorem 1 .

Acknowledgment. We are very grateful to the referee for his many corrections and suggestions.

\section{References}

[1] R. Adler. M. Keane, M. Smorodinsky, A construction of a normal number for the continued fraction transformation. Journal of Number Theory 13 (1981), 95-105.

[2] D. J. ChAmpernowne, The construction of decimals normal in the scale of ten. J. London Math. Soc. 8 (1933), 254-260.

[3] J. Cigler, Asymptotische Verteilung reeller Zahlen mod 1. Monatsh. Math. 64 (1960), 201-225.

[4] M. Drmota, R. F. Tichy, Sequences, Discrepancies and Applications. Lecture Notes in Math, vol. 1651, Springer, 1997.

[5] T. KAMAE, Subsequences of normal sequences. Israel J. Math. 16 (1973), 121-149.

[6] N. M. Korobov, Exponential Sums and their Applications. Kluwer Academic Publishers, Dordrecht, 1992.

[7] L. Kuipers . H. Niederreiter, Uniform Distribution of Sequences. Pure and Applied Mathematics, Wiley-Interscience, New York, 1974.

[8] P. Kirschenhofer, R.F. Tichy, On uniform distribution of double sequences. Manuscripta Math. 35 (1981), 195-207.

[9] M. B. LEVIN, On normal lattice configurations and simultaneously normal numbers. J. Théor. Nombres Bordeaux 13 (2001), 483-527.

[10] M. B. LeVIN, Discrepancy estimate of completely uniform distributed double sequences. In preparation.

[11] M. B. Levin. M. Smorodinsky, $A \mathbb{Z}^{d}$ generalisation of the Davenport-Erdös construction of normal numbers. Colloq. Math. 84/85 (2000), 431-441.

[12] M. B. Levin, M. Smorodinsky, Explicit construction of normal lattice configurations. Colloq. Math. 102 (2005), 33-47.

[13] M. B. Levin. M. Smorodinsky, On polynomial normal lattice configurations. Monatsh. Math. (2005)

[14] A. G. Postnikov, Arithmetic modeling of random processes. Proc. Steklov. Inst. Math. 57 (1960), $84 \mathrm{pp}$.

[15] M. SMORODINSKY. B. WeIss, Normal sequences for Markov shifts and intrinsically ergodic subshifts. Israel J. Math. 59 (1987), 225-233.

[16] B. WeIss, Normal sequences as collectives. Proc. Symp. on Topological Dynamics and Ergodic Theory, Univ. of Kentucky, 1971. 
Mordechay B. LEviN

Department of Mathematics

Bar-Ilan University

52900, Ramat-Gan, Israel

E-mail : mlevin@math.biu.ac.il

Meir SMORODINSKY

School of Mathematical Sciences

Tel Aviv University

69978, Tel-Aviv, Israel

E-mail : meir@math.tau.ac.il 